\title{
Rational KdV Potentials and Differential Galois Theory
}

\author{
Sonia JIMÉNEZ ${ }^{\dagger}$, Juan J. MORALES-RUIZ ${ }^{\ddagger}$, Raquel SÁNCHEZ-CAUCE ${ }^{\S}$ \\ and María-Ángeles ZURRO§ \\ $\dagger$ Junta de Castilla y León, Salamanca, Spain \\ E-mail: sonia.jimver@educa.jcyl.es \\ ‡ Departamento de Matemática Aplicada, E.T.S. Edificación, \\ Universidad Politécnica de Madrid, Madrid, Spain \\ E-mail: juan.morales-ruiz@upm.es \\ $\S$ Departamento de Matemáticas, Universidad Autónoma de Madrid, Madrid, Spain \\ E-mail: raquel.sanchezcauce@predoc.uam.es, mangeles.zurro@uam.es
}

Received September 11, 2018, in final form May 29, 2019; Published online June 25, 2019

https://doi.org/10.3842/SIGMA.2019.047

\begin{abstract}
In this work, using differential Galois theory, we study the spectral problem of the one-dimensional Schrödinger equation for rational time dependent KdV potentials. In particular, we compute the fundamental matrices of the linear systems associated to the Schrödinger equation. Furthermore we prove the invariance of the Galois groups with respect to time, to generic values of the spectral parameter and to Darboux transformations.
\end{abstract}

Key words: differential Galois theory; KdV hierarchy; Schrödinger operator; Darboux transformations; spectral curves; rational solitons

2010 Mathematics Subject Classification: 12H05; 35Q51; 37K10

\section{Introduction}

In 1977 Airault, McKean and Moser studied in [2] some special solutions of the KdV equation,

$$
u_{t}-6 u u_{x}+u_{x x x}=0
$$

like rational and elliptic ones. Then one year later Adler and Moser studied KdV rational solutions of the KdV hierarchy by means of Darboux-Crum transformations, simplifiying the proof of previous results for these solutions [1].

One of the goals of the paper is to study the invariance of the Galois group of the linear system

$$
\begin{aligned}
& \Phi_{x}=U \Phi=\left(\begin{array}{cc}
0 & 1 \\
u-E & 0
\end{array}\right) \Phi, \\
& \Phi_{t_{r}}=V_{r} \Phi=\left(\begin{array}{cc}
G_{r}(u) & F_{r}(u) \\
-H_{r}(u) & -G_{r}(u)
\end{array}\right) \Phi,
\end{aligned}
$$

associated to the KdV hierarchy, with respect to the Darboux transformations and respect to the $\mathrm{KdV}$ flow (i.e., to the time). In fact as a by-product we have obtained more than that: the Galois group is also invariant with respect to generic values of the spectral parameters (see Section 7).

This paper is a contribution to the Special Issue on Algebraic Methods in Dynamical Systems. The full collection is available at https://www.emis.de/journals/SIGMA/AMDS2018.html 
Thus, in some sense this paper can be considered as a continuation of our previous paper [18], where we studied the invariance of the Galois group of the AKNS systems with respect to the Darboux transformations. But one of the essential differences here is that in general we can not use the Darboux invariance result in [18], because the Darboux transformation here is not a well-defined gauge transformation, i.e., it is not inversible. Thus we must use the classical Darboux tranformation of the Schrödinger equation, we call it the Darboux-Crum transform; and then to verify the compatibility of this transform with the complete linear system (1.1).

In Section 3 we study the action of the Darboux transformations over the recursive relations (2.1) inside the KdV hierarchy. We point out that the results in Section 3 hold not only for rational $\mathrm{KdV}$ potentials but also for any arbitrary KdV potential.

Also, in Section 6 we study the action over the spectral curve of the Darboux transformations for stationary KdV arbitrary potentials.

Brezhnev in three papers [5, 6, 7] also consider the Galois groups associated to spectral problem for some KdV potentials. More specifically the so-called finite-gap potentials, where the spectral curve is non-singular. Here we study a completely different situation, where the spectral curves are cuspidal curves, corresponding to Adler-Moser rational type solutions.

In some articles, such as [19] and [25], the authors studied the general Schrödinger equation, i.e., the potential $u$ is a differential indeterminate which satisfies $\mathrm{KdV}_{1}$ equation. This is not our situation here, since we consider the family of Adler-Moser rational potentials in $1+1$ dimensions. We would like to point out that in the stationary case the results in [4] for algebraically integrable systems proved that the Galois group is contained in a torus at each generic point in the spectral curve, when the field of coefficients is a formal field.

However, the general results obtained in Sections 3 and 6 open the door to study more general families of KdV potentials, such as Rosen-Morse potentials or elliptic KdV potentials.

\section{Basic facts on $\mathrm{KdV}$ hierarchy}

Consider the derivations $\partial_{x}, \partial_{t_{1}}, \partial_{t_{2}}, \ldots, \partial_{t_{m}}$ with respect to the variables $x$ and $\boldsymbol{t}=\left(t_{1}, \ldots, t_{m}\right)$. Let $K_{r}$ be a differential field with compatible derivations $\partial_{x}$ and $\partial_{t_{r}}$, with respect to the variables $x$ and $t_{r}$. Let us assume that its field of constants is the field of complex numbers $\mathbb{C}$. Let $E \in \mathbb{C}$ be a complex parameter and $u \in K_{r}$ be a fixed element of $K_{r}$.

Let us consider the differential recursive relations:

$$
f_{0}=1, \quad f_{j, x}=-\frac{1}{4} f_{j-1, x x x}+u f_{j-1, x}+\frac{1}{2} u_{x} f_{j-1},
$$

see [14], where the authors also provided an algorithm to compute $\partial_{x}^{-1}\left(f_{j, x}\right)$. Functions $f_{j}$ are differential polynomials in $u$, see [14, 23]. For the first terms one finds

$$
\begin{aligned}
& f_{0}=1, \quad f_{1}=\frac{1}{2} u+c_{1}, \quad f_{2}=-\frac{1}{8} u_{x x}+\frac{3}{8} u^{2}+\frac{1}{2} c_{1} u+c_{2}, \\
& f_{3}=\frac{1}{32} u_{x x x x}-\frac{5}{16} u u_{x x}-\frac{5}{32} u_{x}^{2}+\frac{5}{16} u^{3}+c_{1}\left(-\frac{1}{8} u_{x x}+\frac{3}{8} u^{2}\right)+\frac{1}{2} c_{2} u+c_{3},
\end{aligned}
$$

for some integration constants $c_{i}$.

It is well known that the time dependent $\mathrm{KdV}$ hierarchy can be constructed as zero curvature condition of the family of integrable systems (see [15, Chapter 1, Section 2]):

$$
\mathfrak{s}_{r} \quad\left\{\begin{array}{l}
\Phi_{x}=U \Phi=\left(\begin{array}{cc}
0 & 1 \\
u-E & 0
\end{array}\right) \Phi \\
\Phi_{t_{r}}=V_{r} \Phi=\left(\begin{array}{cc}
G_{r}(u) & F_{r}(u) \\
-H_{r}(u) & -G_{r}(u)
\end{array}\right) \Phi,
\end{array}\right.
$$


where $F_{r}, G_{r}$ and $H_{r} \in K_{r}$ are differential polynomials of the potential $u$ defined by

$$
\begin{aligned}
& F_{r}=\sum_{j=0}^{r} f_{r-j} E^{j}, \\
& G_{r}=-\frac{F_{r, x}}{2}, \\
& H_{r}=(E-u) F_{r}-G_{r, x}=(E-u) F_{r}+\frac{F_{r, x x}}{2} .
\end{aligned}
$$

Observe that the degree in $E$ of the matrices $V_{r}$ and functions $H_{r}$ is $r+1$. We point out that the first equation of (2.2) is equivalent to the Schrödinger equation

$$
(L-E) \phi=\left(-\partial_{x x}+u-E\right) \phi=0
$$

with $L=-\partial_{x x}+u$.

Its zero curvature condition

$$
U_{t_{r}}-V_{r, x}+\left[U, V_{r}\right]=0
$$

yields to the $\mathrm{KdV}_{r}$ equation

$$
\mathrm{KdV}_{r}: \quad u_{t_{r}}=-\frac{1}{2} F_{r, x x x}-2(E-u) F_{r, x}+u_{x} F_{r} .
$$

Using expressions (2.1) and (2.3), this equation can be rewritten as

$$
\mathrm{KdV}_{r}: \quad u_{t_{r}}=2 f_{r+1, x} .
$$

We recall that the equation (2.8) is called the level $r$ equation of the $\mathrm{KdV}$ hierarchy. Varying $r \in \mathbb{N}$ we get the KdV hierarchy. Whenever we want to specify the dependence on the potential $u$, we will write $f_{j}(u), F_{j}(u), G_{j}(u)$ and $H_{j}(u)$ to emphasize this fact.

\subsection{Adler-Moser rational potentials}

In this section we review the $\mathrm{KdV}_{r}$ rational potentials that Adler and Moser constructed in [1]. These are a family of rational potentials $u_{n}$ for Schrödinger operator $-\partial_{x x}+u$ of the form $u_{n}=-2\left(\log \theta_{n}\right)_{x x}$, where $\theta_{n}$ are functions in the variables $x, t_{r}$ defined by the differential recursion

$$
\theta_{0}=1, \quad \theta_{1}=x, \quad \theta_{n+1, x} \theta_{n-1}-\theta_{n+1} \theta_{n-1, x}=(2 n+1) \theta_{n}^{2}
$$

The solutions of this recursion are polynomials in $x$ with coefficients in the field $F=\mathbb{C}\left(t_{r}\right)$. This is an straighforward consequence of the next result, which is an easy extension of the proof of Lemma 2 in [1].

Lemma 2.1. Let be $F=\mathbb{C}\left(t_{r}\right)$, and $a \in \mathbb{C}^{*}, b \in \mathbb{C}$. Let $\left(F[x], \partial_{x}\right)$ be the ring of polynomials with derivation $\partial_{x}$, whose field of constants is $F$. Let consider the sequence defined recursively by

$$
P_{0}=1, \quad P_{1}=a x+b, \quad P_{n+1, x} P_{n-1}-P_{n+1} P_{n-1, x}=(2 n+1) P_{n}^{2} .
$$

Then $P_{n} \in F[x]$ for all $n$. 
Now, applying Lemma 2.1 for $a=1$ and $b=0$, we obtain that functions $\theta_{n}$ are polynomials of $x$ with coefficients in $\mathbb{C}\left(t_{r}\right)$ for all $n$. We call these polynomials Adler-Moser polynomials.

The first terms of the recursion are

$\begin{array}{cc}n & \theta_{n} \\ 0 & 1 \\ 1 & x \\ 2 & x^{3}+\tau_{2} \\ 3 & x^{6}+5 \tau_{2} x^{3}+\tau_{3} x-5 \tau_{2}^{2}\end{array}$

with $\tau_{j} \in \mathbb{C}\left(t_{r}\right)$ and $\partial_{x} \tau_{j}=0$.

Definition 2.2. The functions

$$
u_{n}:=-2\left(\log \theta_{n}\right)_{x x}
$$

defined by means of Lemma 2.1 are called $K d V$ rational solitons.

Adler and Moser proved in Theorem 2 of [1] that, for each fixed level $r$ of the KdV hierarchy, there exist expressions for $\tau_{j} \in \mathbb{C}\left(t_{r}\right), j=2, \ldots, n$, such that each potential $u_{n}$ defined by means of the formula (2.10) for $\theta_{n}$ is a solution of the $\mathrm{KdV}_{r}$ equation (2.7), for constants $c_{i}=0$, $i=1, \ldots, r$. Hence, the functions $\tau_{2}, \ldots, \tau_{n}$ must be adapted in order to get a solution of the $\mathrm{KdV}_{r}$ equation. When this is the case, i.e., when $u_{n}$ is a solution of the $\mathrm{KdV}_{r}$ equation, we will denote this adjusted potential as $u_{r, n}$ and the corresponding Adler-Moser polynomial as $\theta_{r, n}$ to stress this fact.

Definition 2.3. The functions

$$
u_{r, n}:=-2\left(\log \theta_{r, n}\right)_{x x}
$$

in $\mathbb{C}\left(x, t_{r}\right)$ defined by means of Lemma 2.1 and with the corresponding adjustment of $\tau_{j} \in \mathbb{C}\left(t_{r}\right)$, $j=2, \ldots, n$, are called $K d V_{r}$ rational solitons.

Example 2.4. As an example of adjusted potentials, we show the first Adler-Moser potentials for $r=1$ with the explicit choice of functions $\tau_{2}, \ldots, \tau_{n}$. These potentials are solutions of the $\mathrm{KdV}_{1}$ equation for $c_{1}=0: u_{t_{1}}=\frac{3}{2} u u_{x}-\frac{1}{4} u_{x x x}$. The computations were made using SAGE. We have

$$
\begin{array}{ccc}
n & u_{1, n} & \left(\tau_{2}, \ldots, \tau_{n}\right) \\
0 & 0 & \\
1 & \frac{2}{x^{2}} & \\
2 & \frac{6 x\left(x^{3}-6 t_{1}\right)}{\left(x^{3}+3 t_{1}\right)^{2}} & \left(3 t_{1}\right) \\
3 & \frac{6 x\left(2 x^{9}+675 x^{3} t_{1}^{2}+1350 t_{1}^{3}\right)}{\left(x^{6}+15 x^{3} t_{1}-45 t_{1}^{2}\right)^{2}} & \left(3 t_{1}, 0\right) \\
4 & \frac{10 p_{4}\left(x, t_{1}\right)}{\left(x^{10}+45 x^{7} t_{1}+4725 x t_{1}^{3}\right)^{2}} & \left(3 t_{1}, 0,0\right) \\
5 & \frac{30 x p_{5}\left(x, t_{1}\right)}{\left(\theta_{5}\right)^{2}} & \left(3 t_{1}, 0,0,33075 t_{1}^{3}\right)
\end{array}
$$

where

$$
p_{4}\left(x, t_{1}\right)=2 x^{18}+72 x^{15} t_{1}+2835 x^{12} t_{1}^{2}-66150 x^{9} t_{1}^{3}-1190700 x^{6} t_{1}^{4}+4465125 t_{1}^{6},
$$




$$
\begin{aligned}
p_{5}\left(x, t_{1}\right)= & x^{27}+126 x^{24} t_{1}+7560 x^{21} t_{1}^{2}+5655825 x^{15} t_{1}^{4}+500094000 x^{12} t_{1}^{5} \\
& +4313310750 x^{9} t_{1}^{6}+11252115000 x^{6} t_{1}^{7}+295368018750 x^{3} t_{1}^{8}-590736037500 t_{1}^{9}, \\
\theta_{5}=x^{15}+ & 105 x^{12} t_{1}+1575 x^{9} t_{1}^{2}+33075 x^{6} t_{1}^{3}-992250 x^{3} t_{1}^{4}-1488375 t_{1}^{5} .
\end{aligned}
$$

We notice that the adjustment of $\tau_{i}$ is not linear in $t_{1}$.

\subsection{Spectral curves for $\mathrm{KdV}$ hierarchy}

Next, we consider the stationary KdV hierarchy. Let $u^{(0)}(x)=u\left(x, t_{r}=0\right)$ be an arbitrary stationary potential. The associated linear system, corresponding to system (2.2), will be

$$
\begin{aligned}
& \Phi_{x}=U^{(0)} \Phi=\left(\begin{array}{cc}
0 & 1 \\
u^{(0)}-E & 0
\end{array}\right) \Phi, \\
& \Phi_{t_{r}}=V_{r}^{(0)} \Phi=\left(\begin{array}{cc}
G_{r}\left(u^{(0)}\right) & F_{r}\left(u^{(0)}\right) \\
-H_{r}\left(u^{(0)}\right) & -G_{r}\left(u^{(0)}\right)
\end{array}\right) \Phi .
\end{aligned}
$$

To simplify the notation, from now on we write $F_{r}^{(0)}, G_{r}^{(0)}$ and $H_{r}^{(0)}$ instead of $F_{r}\left(u^{(0)}\right), G_{r}\left(u^{(0)}\right)$ and $H_{r}\left(u^{(0)}\right)$. The zero curvature condition of this system is now the stationary $\operatorname{KdV}_{r}$ equation

$$
\mathrm{s}-\mathrm{KdV}_{r}: \quad 0=-\frac{1}{2} F_{r, x x x}^{(0)}-2\left(E-u^{(0)}\right) F_{r, x}^{(0)}+u_{x}^{(0)} F_{r}^{(0)} .
$$

After applying expressions (2.1) and (2.3), this equation can be rewritten as

$$
\mathrm{s}-\mathrm{KdV}_{r}: \quad 0=2 f_{r+1, x}\left(u^{(0)}\right)=2 f_{r+1, x}^{(0)} .
$$

When the potential $u^{(0)}$ is a solution of the zero curvature condition (2.12) we will say that it is a $\mathrm{s}-\mathrm{KdV}_{r}$ potential. Under this assumption, the spectral curve of system (2.11) for this potential is the characteristic polynomial of matrix $i V_{r}^{(0)}$ :

$$
\begin{aligned}
\Gamma_{r}: \operatorname{det}\left(\mu I_{2}-i V_{r}^{(0)}\right) & =\mu^{2}+\left(G_{r}^{(0)}\right)^{2}-F_{r}^{(0)} H_{r}^{(0)} \\
& =\mu^{2}-\frac{F_{r}^{(0)} F_{r, x x}^{(0)}}{2}+\left(u^{(0)}-E\right)\left(F_{r}^{(0)}\right)^{2}+\frac{\left(F_{r, x}^{(0)}\right)^{2}}{4} \\
& =\mu^{2}-R_{2 r+1}(E)=0 .
\end{aligned}
$$

(see for instance [15] for a general definition of spectral curve). We denote by $p_{r}(E, \mu)=$ $\mu^{2}-R_{2 r+1}(E)$ the equation that defines the spectral curve. We will use the following notation

$$
R_{2 r+1}(E)=\sum_{i=0}^{2 r+1} C_{i} E^{i},
$$

where $C_{i}$ are differential polynomials in $u^{(0)}$ with constant coefficients.

Lemma 2.5. We have the following equality $\partial_{x} C_{0}=-2 f_{r} f_{r+1, x}$.

Proof. Replacing $E=0$ in (2.13) we find

$$
R_{2 r+1}(0)=C_{0}=\frac{-f_{r, x} f_{r, x}}{4}+\frac{f_{r} f_{r, x x}}{2}-u^{(0)} f_{r} f_{r} .
$$

By derivating with respect to $x$ and using formula (2.1) we arrive to the required expression. 
With this matrix presentation it is easy to prove the following result due to Burchnall and Chaundy [8]:

Proposition 2.6 ([8, Section II, p. 560]). Let $u=u(x)$ be solution of equation (2.12), we have that $p(E, \mu)=\mu^{2}-R_{2 r+1}(E) \in \mathbb{C}[\mu, E]$. Moreover, $R_{2 r+1}(E)$ is a polynomial of degree $2 r+1$ in $\mathbb{C}[E]$.

Remark 2.7. A potential $u$ can be a solution of several equations of the KdV hierarchy. Therefore, for each level considered, there would be a different spectral curve for the same potential. This ambiguity is clarified when the corresponding Schrodinger operator's centralizer is considered. Furthermore, this centralizer is isomorphic to the ring of rational functions of an algebraic plane curve: the spectral curve that corresponds to the first level of the hierarchy of which the potential $u$ is a solution. See [22].

This proposition together with Lemma 2.5 and relation (2.1) yields to the following result.

Corollary 2.8. Let $\mu^{2}-R_{2 r+1}(E)=0$ be the spectral curve for potential $u^{(0)}$. If the degree of $R_{2 r+1}(E)$ is $2 r+1$ in $E$ then, $u^{(0)}$ is solution of a $s-K d V_{r}$ equation.

Now, we consider the Adler-Moser potentials $u_{r, n}$. We have the following result in the stationary case [1]:

Lemma 2.9. For $\tau_{j}=0, j=2, \ldots, n$, the Adler-Moser polynomials and potentials become

$$
\theta_{n}^{(0)}(x)=\theta_{n}(x, 0)=x^{n(n+1) / 2} \quad \text { and } \quad u_{r, n}^{(0)}(x)=u_{r, n}\left(x, t_{r}=0\right)=n(n+1) x^{-2} .
$$

For a fixed $n$, potential $u_{r, n}^{(0)}(x)=n(n+1) x^{-2}$ defined in the aforementioned lemma is solution of the level $n$ equation of the stationary $\mathrm{KdV}$ hierarchy, the s-KdV $\mathrm{K}_{n}$ equation. This implies that in the stationary case we will have $r=n$, i.e., for these s-KdV potentials the iteration level of the recursion (2.9) is the same as the s-KdV level. For this reason, from now on we will denote the stationary Adler-Moser potentials just by $u_{n}^{(0)}(x)$ and we will refer to level $n$ stationary KdV equation (instead of level $r$ ):

$$
\mathrm{s}-\mathrm{KdV}_{n}: \quad 0=2 f_{n+1, x}^{(0)} .
$$

It is well known that the spectral curve associated to system (2.11) for these Adler-Moser stationary potentials are

$$
\Gamma_{n}: p_{n}(E, \mu)=\mu^{2}-E^{2 n+1}=0 .
$$

Therefore, we will associate these curves corresponding to the stationary situation, to system (2.2) for Adler-Moser potentials $u_{r, n}$.

Remark 2.10. If we take the potential $u_{r, n}$ solution of $\operatorname{KdV}_{r}$ equation, then the potential $u_{n}^{(0)}(x)$ is a solution of the s-KdV $\mathrm{K}_{n}$ equation. Thus, we can link the level $r$ of the time-dependent $\mathrm{KdV}$ hierarchy with the level $n$ of the stationary KdV hierarchy.

\section{Darboux transformations for $f_{j}$}

In this section we establish a series of results that will allow us to perform Darboux transformations to $\mathrm{KdV}$ differential systems (2.2) in the case we have particular solutions at energy level zero. In this way, we can extend the techniques to compute matrix Darboux transformations developed, for instance, in [16] to the only case where they are not valid: $E=0$. 
For that, we will consider the classical Darboux-Crum transformations for the Schrödinger equation and we will present the behaviour of these transformations acting on the differential polynomials $f_{j}(u)$.

Let us consider the Schrödinger equation

$$
\left(L-E_{0}\right) \phi=\left(-\partial_{x x}+u-E_{0}\right) \phi=0
$$

where $E_{0}$ is a fixed energy level. Let $\phi_{0}$ be a solution of such equation. Recall that a Darboux transformation of a function $\phi$ by $\phi_{0}$ is defined by the formula

$$
\mathrm{DT}\left(\phi_{0}\right) \phi=\phi_{x}-\frac{\phi_{0, x}}{\phi_{0}} \phi
$$

Then the transformed function $\widetilde{\phi}=\operatorname{DT}\left(\phi_{0}\right) \phi$ is a solution of the Schrödinger equation for potential $\widetilde{u}=u-2\left(\log \phi_{0}\right)_{x x}$, whenever $\phi$ is a solution of Schrödinger equation for potential $u$ and energy level $E \neq E_{0}[10,11,12,20]$. We will denote by $\mathrm{DT}\left(\phi_{0}\right) u$ the potential $\widetilde{u}$ to point out the fact that it depends on the choice of $\phi_{0}$.

Next we can observe that the Riccati equation

$$
\sigma_{x}=u-E_{0}-\sigma^{2}
$$

has $\sigma_{0}=\left(\log \phi_{0}\right)_{x}$ as solution, and then

$$
\operatorname{DT}\left(\phi_{0}\right) u=u-2 \sigma_{0, x} .
$$

In this way, we retrieve a Riccati equation for $\widetilde{u}$ :

$$
\widetilde{u}=u-2 \sigma_{x}=\left(\sigma_{x}+E_{0}+\sigma^{2}\right)-2 \sigma_{x}=\sigma^{2}-\sigma_{x}+E_{0} .
$$

Moreover, whenever we have a solution $\phi$ of the Schrödinger equation (2.6), the formula $\sigma=$ $(\log \phi)_{x}$ gives a solution of the Riccati equation (3.2). Hence, $\sigma$ satisfies the nonlinear differential equation

$$
\sigma_{x x}=u_{x}-2 \sigma \sigma_{x}
$$

Next, we consider the matrix differential system (2.2). Then we perform a Darboux transformation, $\operatorname{DT}\left(\phi_{0}\right)$, on it obtaing a new differential system, say $\Phi_{x}=\widetilde{U} \Phi, \Phi_{t_{r}}=\widetilde{V}_{r} \Phi$, whose zero curvature condition is still equation (2.7). Let $F_{r}(\widetilde{u}), G_{r}(\widetilde{u})$ and $H_{r}(\widetilde{u})$ be the corresponding entries of the matrix $\widetilde{V}_{r}$. These differential polynomials are given by expressions (2.3), (2.4) and $(2.5)$ in terms on $f_{j}(\widetilde{u})$. We will establish the relation between $f_{j}(\widetilde{u})$ and $f_{j}(u)$ in the next theorem.

Theorem 3.1. Let $\phi$ be a solution of Schrödinger equation (3.1). Let be $\sigma=(\log \phi)_{x}$ and $\widetilde{u}=u-2 \sigma_{x}$ the Darboux transformed of $u$ by $\phi$. Then, we have

$$
f_{j}(\widetilde{u})=f_{j}(u)+A_{j}, \quad \text { for } \quad j=0,1,2, \ldots,
$$

where $A_{j}$ is a differential polynomial in $u$ and $\sigma$. Moreover, $A_{j}$ satisfies the recursive differential relations

1) $A_{j}=-\frac{1}{4} A_{j-1, x x}+u A_{j-1}-\frac{3}{2} \sigma_{x} A_{j-1}-\sigma_{x} f_{j-1}(u)$ and

2) $A_{j, x}+2 \sigma A_{j}+2 f_{j, x}(u)=0$. 
Proof. We will proceed by induction on $n$.

First, we prove by induction that $f_{j}(\widetilde{u})=f_{j}(u)+A_{j}$. For $j=0$ we have $f_{0}(\widetilde{u})=1=$ $f_{0}(u)+A_{0}$, where $A_{0}=0$. We suppose it is true for $j$ and we prove it for $j+1$. By applying equation (2.1) and induction hypothesis we find

$$
\begin{aligned}
f_{j+1, x}(\widetilde{u})= & -\frac{1}{4} f_{j, x x x}(\widetilde{u})+\widetilde{u} f_{j, x}(\widetilde{u})+\frac{1}{2} \widetilde{u}_{x} f_{j}(\widetilde{u}) \\
= & -\frac{1}{4} f_{j, x x x}(u)+u f_{j, x}(u)+\frac{1}{2} u_{x} f_{j}(u)-\frac{1}{4} A_{j, x x x}+u A_{j, x}-2 f_{j, x}(u) \sigma_{x} \\
& -2 A_{j, x} \sigma_{x}+\frac{1}{2} u_{x} A_{j}-f_{j}(u) \sigma_{x x}-A_{j} \sigma_{x x}=f_{j+1, x}(u)+A_{j+1, x},
\end{aligned}
$$

for

$$
A_{j+1, x}=-\frac{A_{j, x x x}}{4}+u A_{j, x}-2 f_{j, x}(u) \sigma_{x}-2 A_{j, x} \sigma_{x}+\frac{u_{x} A_{j}}{2}-f_{j}(u) \sigma_{x x}-A_{j} \sigma_{x x} .
$$

Thus, $f_{j+1}(\widetilde{u})=f_{j+1}(u)+A_{j+1}$ as we wanted to prove.

Now, we prove statements 1 and 2 . We do it by induction and simultaneously. Since $A_{0}=0$ and $f_{0}(u)=f_{0}(\widetilde{u})=1$, the case $j=0$ is the trivial one. So, we start the induction process in $j=1$. For this, by using recursion formula (2.1) we have

$$
f_{1, x}(\widetilde{u})=-\frac{1}{4} f_{0, x x x}(\widetilde{u})+\widetilde{u} f_{0, x}(\widetilde{u})+\frac{1}{2} \widetilde{u}_{x} f_{0}(\widetilde{u})=\frac{1}{2} \widetilde{u}_{x} .
$$

Hence, $f_{1}(\widetilde{u})=\frac{\widetilde{u}}{2}+c_{1}=\frac{u}{2}-\sigma_{x}+c_{1}=f_{1}(u)-\sigma_{x}$, then $A_{1}=-\sigma_{x}$. For $j=1$ statements 1 and 2 read

$$
\begin{aligned}
& \text { 1) }-\frac{1}{4} A_{0, x x}+u A_{0}-\frac{3}{2} \sigma_{x} A_{0}-\sigma_{x} f_{0}(u)=-\sigma_{x}=A_{1} \text { and } \\
& \text { 2) }-2 f_{1, x}(u)-A_{1, x}=-u_{x}+\sigma_{x x}=-2 \sigma \sigma_{x}=2 \sigma A_{1},
\end{aligned}
$$

by equation (3.4). Now, we suppose the both statements are true for $j$ and we prove them for $j+1$. Derivation with respect to $x$ in the right hand side of statement 1 yields to

$$
\begin{aligned}
& -\frac{A_{j, x x x}}{4}+u_{x} A_{j}+u A_{j, x}-\frac{3}{2} \sigma_{x x} A_{j}-\frac{3}{2} \sigma_{x} A_{j, x}-\sigma_{x x} f_{j}(u)-\sigma_{x} f_{j, x}(u) \\
& =-\frac{A_{j, x x x}}{4}+u A_{j, x}-\sigma_{x x} f_{j}(u)-\sigma_{x x} A_{j}-\frac{\sigma_{x x} A_{j}}{2}+u_{x} A_{j}-\frac{3}{2} \sigma_{x} A_{j, x}-\sigma_{x} f_{j, x}(u) .
\end{aligned}
$$

Applying equality (3.4) to the term $\sigma_{x x} A_{j} / 2$ we get

$$
\begin{aligned}
& -\frac{A_{j, x x x}}{4}+u A_{j, x}-\sigma_{x x} f_{j}(u)-\sigma_{x x} A_{j}-\frac{u_{x} A_{j}-2 \sigma \sigma_{x} A_{j}}{2}+u_{x} A_{j}-\frac{3}{2} \sigma_{x} A_{j, x}-\sigma_{x} f_{j, x}(u) \\
& =-\frac{A_{j, x x x}}{4}+u A_{j, x}-\sigma_{x x} f_{j}(u)-\sigma_{x x} A_{j}+\sigma \sigma_{x} A_{j}+\frac{u_{x} A_{j}}{2}-\frac{3}{2} \sigma_{x} A_{j, x}-\sigma_{x} f_{j, x}(u) \\
& =-\frac{A_{j, x x x}}{4}+u A_{j, x}-\sigma_{x x} f_{j}(u)-\sigma_{x x} A_{j}+\frac{u_{x} A_{j}}{2}-2 \sigma_{x} A_{j, x}-\sigma_{x} f_{j, x}(u)+\sigma_{x}\left(\sigma A_{j}+\frac{1}{2} A_{j, x}\right) .
\end{aligned}
$$

Applying induction hypothesis for statement 2 we have

$$
\begin{gathered}
-\frac{A_{j, x x x}}{4}+u A_{j, x}-\sigma_{x x} f_{j}(u)-\sigma_{x x} A_{j}+\frac{u_{x} A_{j}}{2}-2 \sigma_{x} A_{j, x}-\sigma_{x} f_{j, x}(u)-\sigma_{x} f_{j, x}(u) \\
=-\frac{A_{j, x x x}}{4}+u A_{j, x}-\sigma_{x x} f_{j}(u)-\sigma_{x x} A_{j}+\frac{u_{x} A_{j}}{2}-2 \sigma_{x} A_{j, x}-2 \sigma_{x} f_{j, x}(u)
\end{gathered}
$$


which is exactly expression (3.5) for $A_{j+1, x}$. So, we can assume that

$$
A_{j+1}=-\frac{A_{j, x x}}{4}+u A_{j}-\frac{3}{2} \sigma_{x} A_{j}-\sigma_{x} f_{j}(u) .
$$

Thus, statement 1 is proved.

Finally, by equations (2.1), (3.5), (3.4) and induction hypothesis we find for statement 2

$$
\begin{aligned}
-2 f_{j+1, x}-A_{j+1, x}= & \frac{f_{j, x x x}(u)}{2}-2 u f_{j, x}(u)-u_{x} f_{j}(u)+\frac{A_{j, x x x}}{4}-u A_{j, x}+2 f_{j, x}(u) \sigma_{x} \\
& -\frac{u_{x} A_{j}}{2}+2 A_{j, x} \sigma_{x}+f_{j}(u) \sigma_{x x}+A_{j} \sigma_{x x} \\
= & \left(\frac{f_{j, x}(u)}{2}+\frac{A_{j, x}}{4}\right)_{x x}+\left(-2 f_{j, x}(u)-A_{j, x}\right)\left(u-\sigma_{x}\right)-u_{x} f_{j}(u) \\
& -\frac{u_{x} A_{j}}{2}+A_{j, x} \sigma_{x}+f_{j}(u) \sigma_{x x}+A_{j} \sigma_{x x} \\
= & -\frac{\sigma A_{j, x x}}{2}+2 u \sigma A_{j}+A_{j}\left(\frac{\sigma_{x x}}{2}-\frac{u_{x}}{2}-2 \sigma \sigma_{x}\right)+f_{j}(u)\left(\sigma_{x x}-u_{x}\right) \\
= & -\frac{\sigma A_{j, x x}}{2}+2 u \sigma A_{j}-3 A_{j} \sigma \sigma_{x}-2 f_{j}(u) \sigma \sigma_{x} \\
= & 2 \sigma\left(-\frac{A_{j, x x}}{4}+u A_{j}-\frac{3}{2} \sigma_{x} A_{j}-\sigma_{x} f_{j}(u)\right)=2 \sigma A_{j+1}
\end{aligned}
$$

by statement 1 . Therefore, statement 2 is also proved. This completes the proof.

Example 3.2. To illustrate the previous theorem we will consider the following $\mathrm{KdV}_{2}$ potentials in the system (2.2).

Let us take

$$
u=\frac{6\left(2 x^{10}+270 x^{5} t_{2}+675 t_{2}^{2}\right)}{x^{2}\left(x^{5}-45 t_{2}\right)^{2}}
$$

and the solution $\phi_{0}=\frac{x^{2}}{x^{5}-45 t_{2}}$. Then $\widetilde{u}=\frac{6}{x^{2}}$. Observe that

$$
f_{1}(u)=\frac{u}{2}=\frac{3\left(2 x^{10}+270 x^{5} t_{2}+675 t_{2}^{2}\right)}{x^{2}\left(x^{5}-45 t_{2}\right)^{2}}, \quad f_{2}(u)=-\frac{u_{x x}}{8}+\frac{3}{8} u^{2}=\frac{45 x\left(x^{5}+30 t_{2}\right)}{\left(x^{5}-45 t_{2}\right)^{2}},
$$

and also

$$
f_{1}(\widetilde{u})=\frac{\widetilde{u}}{2}=\frac{3}{x^{2}}, \quad f_{2}(\widetilde{u})=-\frac{\widetilde{u}_{x x}}{8}+\frac{3}{8} \widetilde{u}^{2}=\frac{9}{x^{4}} .
$$

Hence, in this case

$$
\begin{aligned}
& A_{1}=f_{1}(\widetilde{u})-f_{1}(u)=\frac{-3\left(x^{10}+360 x^{5} t_{2}-1350 t_{2}^{2}\right)}{x^{2}\left(x^{5}-45 t_{2}\right)^{2}}, \\
& A_{2}=f_{2}(\widetilde{u})-f_{2}(u)=\frac{-9\left(4 x^{10}+240 x^{5} t_{2}-2025 t_{2}^{2}\right)}{x^{4}\left(x^{5}-45 t_{2}\right)^{2}} .
\end{aligned}
$$

By a direct computation we can verify that the $A_{j}$ satisfy the relations 1 and 2 of Theorem 3.1.

Corollary 3.3. For $i \geq j$ we have the following equality

$$
\sum_{j=0}^{i}\left(2 \sigma A_{i-j}+2 f_{i-j, x}(u)+A_{i-j, x}\right) E^{j}=0
$$


Theorem 3.1 has several interesting consequences. The main ones are the relations that the transformed potential $\widetilde{u}$ produce for functions $F_{r}(u)$. Next we stablish some of them, which will be used in the following sections. In particular, Proposition 3.5 is specially interesting since it gives a relation between $\sigma_{x}$ and $\sigma_{t_{r}}$.

Proposition 3.4. Let $A_{i}$ and $\sigma$ be as in Theorem 3.1. For $i=0,1,2, \ldots$ we have

1. $F_{i}(\widetilde{u})=F_{i}(u)+P_{i}$, where $P_{i}=\sum_{j=0}^{i} E^{j} A_{i-j}$.

2. Moreover $P_{i, x}+2 \sigma P_{i}+2 F_{i, x}(u)=0$.

Proof. It is an immediate consequence of Theorem 3.1.

Proposition 3.5. Let $u$ be a solution of $K d V_{r}$ equation. Let $\phi$ be a solution of Schrödinger equation (2.6) for potential $u$ and energy $E_{0}$. Let be $\sigma=(\log \phi)_{x}$. Consider $A_{r+1}$ as defined in Theorem 3.1 and $P_{r}$ as defined in Proposition 3.4. Then, we have

$$
\sigma_{t_{r}}=-A_{r+1}=\frac{1}{4} P_{r, x x}+E P_{r}+\sigma_{x} F_{r}(u)+\frac{1}{2} P_{r}\left(-2 u+3 \sigma_{x}\right) .
$$

Proof. We compare the zero curvature conditions for $u$ and $\widetilde{u}$ :

$$
\begin{aligned}
& u_{t_{r}}=2 f_{r+1, x}(u)=-\frac{1}{2} F_{r, x x x}(u)+2(u-E) F_{r, x}(u)+u_{x} F_{r}(u), \\
& \widetilde{u}_{t_{r}}=2 f_{r+1, x}(\widetilde{u})=-\frac{1}{2} F_{r, x x x}(\widetilde{u})+2(\widetilde{u}-E) F_{r, x}(\widetilde{u})+\widetilde{u}_{x} F_{r}(\widetilde{u}) .
\end{aligned}
$$

We prove the first equality. For this, we have

$$
\widetilde{u}_{t_{r}}=\left(u-2 \sigma_{x}\right)_{t_{r}}=u_{t_{r}}-2 \sigma_{x, t_{r}} \quad \text { and } \quad 2 f_{r+1, x}(\widetilde{u})=2 f_{r+1, x}(u)+2 A_{r+1, x}
$$

by Theorem 3.1. Then

$$
2 \sigma_{x, t_{r}}=u_{t_{r}}-\widetilde{u}_{t_{r}}=2 f_{r+1, x}(u)-2 f_{r+1, x}(\widetilde{u})=-2 A_{r+1, x} .
$$

Thus, $\sigma_{t_{r}}=-A_{r+1}$.

Now, we prove the second equality. Using expression (3.3) for $\widetilde{u}$ and applying 3.4 (1), we obtain

$$
\begin{aligned}
\widetilde{u}_{t_{r}}= & -\frac{1}{2} F_{r, x x x}(u)+2(u-E) F_{r, x}(u)+u_{x} F_{r}(u)-\frac{1}{2} P_{r, x x x}-2(E-u) P_{r, x} \\
& -4 \sigma_{x} F_{r, x}(u)-4 \sigma_{x} P_{r, x}+u_{x} P_{r}-2 \sigma_{x x} F_{r}(u)-2 \sigma_{x x} P_{r} .
\end{aligned}
$$

Since $2 \sigma_{x, t_{r}}=u_{t_{r}}-\widetilde{u}_{t_{r}}$, we have

$$
2 \sigma_{x, t_{r}}=\frac{1}{2} P_{r, x x x}+2 E P_{r, x}-2 u P_{r, x}+4 \sigma_{x} F_{r, x}(u)+4 \sigma_{x} P_{r, x}-u_{x} P_{r}+2 \sigma_{x x} F_{r}(u)+2 \sigma_{x x} P_{r} .
$$

Applying (2) of Proposition 3.4 to the expresion $\sigma_{x} P_{r, x}$, we find

$$
\begin{aligned}
2 \sigma_{x, t_{r}}= & \frac{1}{2} P_{r, x x x}+2 E P_{r, x}-2 u P_{r, x}+4 \sigma_{x} F_{r, x}(u)+3 \sigma_{x} P_{r, x}+\sigma_{x}\left(-2 \sigma P_{r}-2 F_{r, x}(u)\right) \\
& -u_{x} P_{r}+2 \sigma_{x x} F_{r}(u)+2 \sigma_{x x} P_{r} \\
= & \frac{1}{2} P_{r, x x x}+2 E P_{r, x}+2\left(\sigma_{x x} F_{r}(u)+\sigma_{x} F_{r, x}(u)\right)+P_{r, x}\left(-2 u+3 \sigma_{x}\right) \\
& +P_{r}\left(-2 \sigma \sigma_{x}-u_{x}+2 \sigma_{x x}\right) .
\end{aligned}
$$


Moreover, for the coefficient of $P_{r}$ we have

$$
-2 \sigma \sigma_{x}-u_{x}+2 \sigma_{x x}=\left(-\sigma^{2}-u+2 \sigma_{x}\right)_{x}=\left(-2 u+3 \sigma_{x}\right)_{x}
$$

by (3.2). Thus, we obtain

$$
2 \sigma_{x, t_{r}}=\left(\frac{1}{2} P_{r, x x}+2 E P_{r}+2 \sigma_{x} F_{r}(u)+P_{r}\left(-2 u+3 \sigma_{x}\right)\right)_{x} .
$$

Therefore, we have proved the statement.

We finish this section with the following technical result. It makes a connection between differential polynomials $f_{r}(u)$ and some differential polynomials $g_{r}(\sigma)$ defined by

$$
g_{r}(\sigma):=-A_{r+1}=\frac{1}{2} P_{r, x x}+2 E P_{r}+2 \sigma_{x} F_{r}(u)+P_{r}\left(-2 u+3 \sigma_{x}\right) .
$$

Proposition 3.6. We have the following relations:

1) $\left(2 \sigma+\partial_{x}\right) g_{r}(\sigma)=2 f_{r+1, x}(u)=-\frac{1}{2} F_{r, x x x}(u)+2(u-E) F_{r, x}(u)+u_{x} F_{r}(u)$ and

2) $\left(2 \sigma-\partial_{x}\right) g_{r}(\sigma)=2 f_{r+1, x}(\widetilde{u})=-\frac{1}{2} F_{r, x x x}(\widetilde{u})+2(\widetilde{u}-E) F_{r, x}(\widetilde{u})+\widetilde{u}_{x} F_{r}(\widetilde{u})$.

Proof. The statement 1 is the statement 2 of Theorem 3.1 rewritten. For statement 2 we have

$$
\begin{aligned}
2 f_{r+1, x}(\widetilde{u}) & =2 f_{r+1, x}(u)+2 A_{r+1, x}=2 \sigma g_{r}(\sigma)+g_{r, x}(\sigma)-2 g_{r, x}(\sigma)=2 \sigma g_{r}(\sigma)-g_{r, x}(\sigma) \\
& =\left(2 \sigma-\partial_{x}\right) g_{r}(\sigma)
\end{aligned}
$$

by statement 1 and equation (3.7).

\section{Fundamental matrices for $\mathrm{KdV}_{r}$ rational Schrödinger operators}

In this section we obtain a fundamental matrix for the system (2.2) depending on the energy level $E$. The spectral curve is the tool that will allow us to understand why fundamental matrices present different behaviours according to the values of the energy.

For stationary rational potentials $u_{n}^{(0)}=n(n+1) x^{-2}$, it is well known that the spectral curve associated to the following system

$$
\begin{aligned}
& \Phi_{x}=U^{(0)} \Phi=\left(\begin{array}{cc}
0 & 1 \\
u_{n}^{(0)}-E & 0
\end{array}\right) \Phi, \\
& \Phi_{t_{n}}=V_{n}^{(0)} \Phi=\left(\begin{array}{cc}
G_{n}\left(u_{n}^{(0)}\right) & F_{n}\left(u_{n}^{(0)}\right) \\
-H_{n}\left(u_{n}^{(0)}\right) & -G_{n}\left(u_{n}^{(0)}\right)
\end{array}\right) \Phi
\end{aligned}
$$

is the algebraic plane curve in $\mathbb{C}^{2}$ given by

$$
\Gamma_{n}: p_{n}(\mu, E)=\mu^{2}-E^{2 n+1}=0 .
$$

Whenever an Adler-Moser potential $u_{r, n}(x, t)$ is time dependent, we will consider $\Gamma_{n}$ as the spectral curve associated to its corresponding linear differential system (2.2). Observe that $(E, \mu)=(0,0)$ is the unique affine singular point of $\Gamma_{n}$. It turns out that for $E \neq 0$ the behaviour of the fundamental matrix associated to the system

$$
\Phi_{x}=U \Phi=\left(\begin{array}{cc}
0 & 1 \\
u_{r, n}-E & 0
\end{array}\right) \Phi
$$




$$
\Phi_{t_{r}}=V_{r} \Phi=\left(\begin{array}{cc}
-\frac{F_{r, x}\left(u_{r, n}\right)}{2} & F_{r}\left(u_{r, n}\right) \\
\left(u_{r, n}-E\right) F_{r}\left(u_{r, n}\right)-\frac{F_{r, x x}\left(u_{r, n}\right)}{2} & \frac{F_{r, x}\left(u_{r, n}\right)}{2}
\end{array}\right) \Phi
$$

presents the same algebraic structure since the point $P=(E, \mu)$ is a regular point of $\Gamma_{n}$. A fundamental matrix for $E=0$ can be also computed. However, it is not obtained by a specialization process from the fundamental matrix obtained for a regular point. We include some examples in this section.

\subsection{Fundamental matrices for $E=0$}

In this section, we compute explicitly fundamental matrices of system (2.2) when the potential $u$ is $u_{r, n}=-2\left(\log \theta_{r, n}\right)_{x x}$ and $E=0$. Recall that $u_{r, n}$ is a solution of $\operatorname{KdV}_{r}$. Hence, we study the system

$$
\begin{aligned}
& \Phi_{x}=U \Phi=\left(\begin{array}{cc}
0 & 1 \\
u_{r, n} & 0
\end{array}\right) \Phi, \\
& \Phi_{t_{r}}=V_{r} \Phi=\left(\begin{array}{cc}
-\frac{f_{r, x}\left(u_{r, n}\right)}{2} & f_{r}\left(u_{r, n}\right) \\
u_{r, n} f_{r}\left(u_{r, n}\right)-\frac{f_{r, x x}\left(u_{r, n}\right)}{2} & \frac{f_{r, x}\left(u_{r, n}\right)}{2}
\end{array}\right) \Phi .
\end{aligned}
$$

It is obvious that the zero curvature condition of this system is the $\mathrm{KdV}_{r}$ equation for $c_{i}=0$, $i=1, \ldots, r$ :

$$
\partial_{t_{r}}\left(u_{r, n}\right)=2 f_{r+1, x}\left(u_{r, n}\right) .
$$

From now on we will denote $u_{r, n, t_{r}}=\partial_{t_{r}}\left(u_{r, n}\right)$.

We have the following result:

Theorem 4.1. Let $n$ be a non negative integer. For $E=0$ and $u=u_{r, n}$, a fundamental matrix for system (4.2) is

$$
\mathcal{B}_{n, 0}^{(r)}=\left(\begin{array}{cc}
\phi_{1, r, n} & \phi_{2, r, n} \\
\phi_{1, r, n, x} & \phi_{2, r, n, x}
\end{array}\right)
$$

where

$$
\phi_{1, r, n}\left(x, t_{r}, 0\right)=\frac{\theta_{r, n-1}}{\theta_{r, n}} \quad \text { and } \quad \phi_{2, r, n}\left(x, t_{r}, 0\right)=\frac{\theta_{r, n+1}}{\theta_{r, n}} .
$$

For $n=0$ we define $\theta_{r,-1}:=1$. We notice that $\phi_{2, r, n}=\left(\phi_{1, r, n+1}\right)^{-1}$.

Proof. We prove it by induction on $n$. For $n=0$ the definition $\theta_{r, 0}=1$ gives $u_{r, 0}=0$. So, the system (4.2) reads

$$
\begin{aligned}
\left(\begin{array}{cc}
\phi_{1, r, 0, x} & \phi_{2, r, 0, x} \\
\phi_{1, r, 0, x x} & \phi_{2, r, 0, x x}
\end{array}\right) & =\left(\begin{array}{ll}
0 & 1 \\
0 & 0
\end{array}\right)\left(\begin{array}{cc}
\phi_{1, r, 0} & \phi_{2, r, 0} \\
\phi_{1, r, 0, x} & \phi_{2, r, 0, x}
\end{array}\right)=\left(\begin{array}{cc}
\phi_{1, r, 0, x} & \phi_{2, r, 0, x} \\
0 & 0
\end{array}\right), \\
\left(\begin{array}{cc}
\phi_{1, r, 0, t_{r}} & \phi_{2, r, 0, t_{r}} \\
\phi_{1, r, 0, x t_{r}} & \phi_{2, r 0, x t_{r}}
\end{array}\right) & =\left(\begin{array}{ll}
0 & 0 \\
0 & 0
\end{array}\right)\left(\begin{array}{cc}
\phi_{1, r, 0} & \phi_{2, r, 0} \\
\phi_{1, r, 0, x} & \phi_{2, r, 0, x}
\end{array}\right)=\left(\begin{array}{ll}
0 & 0 \\
0 & 0
\end{array}\right) .
\end{aligned}
$$

Thus, $\phi_{1, r, 0}=1$ and $\phi_{2, r, 0}=x$ generate $\mathcal{B}_{0,0}^{(r)}$. Since $\theta_{r, 1}=x$ we have that $\phi_{1, r, 0}=\frac{\theta_{r,-1}}{\theta_{r, 0}}$ and $\phi_{2, r, 0}=\frac{\theta_{r, 1}}{\theta_{r, 0}}$. 
Now, we suppose the statement is true for $n$ and prove it for $n+1$. For $n$ we know that $\phi_{1, r, n}=\frac{\theta_{r, n-1}}{\theta_{r, n}}$ and $\phi_{2, r, n}=\frac{\theta_{r, n+1}}{\theta_{r, n}}$ generate $\mathcal{B}_{n, 0}^{(r)}$. Therefore, $\phi_{1, r, n}$ and $\phi_{2, r, n}$ are solutions of Schrödinger equation $\phi_{x x}=u_{r, n} \phi$. We apply a Darboux transformation with $\phi_{2, r, n}$ to this Schrödinger equation and we obtain

$$
\begin{aligned}
\operatorname{DT}\left(\phi_{2, r, n}\right) u_{r, n} & =u_{r, n}-2\left(\log \phi_{2, r, n}\right)_{x x}=-2\left(\log \theta_{r, n}\right)_{x x}-2\left(\log \phi_{2, r, n}\right)_{x x} \\
& =-2\left(\log \phi_{2, r, n} \theta_{r, n}\right)_{x x}=-2\left(\log \theta_{r, n+1}\right)_{x x}=u_{r, n+1}, \\
\operatorname{DT}\left(\phi_{2, r, n}\right) \phi_{1, r, n} & =\phi_{1, r, n, x}-\frac{\phi_{2, r, n, x}}{\phi_{2, r, n}} \phi_{1, r, n}=-(2 n+1) \frac{\theta_{n}}{\theta_{r, n+1}}=-(2 n+1) \phi_{1, r, n+1} .
\end{aligned}
$$

So, $\phi_{1, r, n+1}=\frac{\theta_{r, n}}{\theta_{r, n+1}}$ is a solution of $\phi_{x x}=u_{r, n+1} \phi$ and, obviously, $\left(\phi_{1, r, n+1}, \phi_{1, r, n+1, x}\right)^{t}$ is a column solution of the first equation of the system for $u_{r, n+1}$.

Now we verify that this column matrix is also a solution of the second equation

$$
\begin{aligned}
& \left(\begin{array}{c}
\phi_{1, r, n+1, t_{r}} \\
\phi_{1, r, n+1, x t_{r}}
\end{array}\right)=\left(\begin{array}{cc}
-\frac{f_{r, x}\left(u_{r, n+1}\right)}{2} & f_{r}\left(u_{r, n+1}\right) \\
u_{r, n+1} f_{r}\left(u_{r, n+1}\right)-\frac{f_{r, x x}\left(u_{r, n+1}\right)}{2} & \frac{f_{r, x}\left(u_{r, n+1}\right)}{2}
\end{array}\right)\left(\begin{array}{c}
\phi_{1, r, n+1} \\
\phi_{1, r, n+1, x}
\end{array}\right) \\
& =\left(\begin{array}{c}
-\frac{f_{r, x}\left(u_{r, n+1}\right)}{2} \phi_{1, r, n+1}+f_{r}\left(u_{r, n+1}\right) \phi_{1, r, n+1, x} \\
\left(u_{r, n+1} f_{r}\left(u_{r, n+1}\right)-\frac{f_{r, x x}\left(u_{r, n+1}\right)}{2}\right) \phi_{1, r, n+1}+\frac{f_{r, x}\left(u_{r, n+1}\right)}{2} \phi_{1, r, n+1, x}
\end{array}\right) .
\end{aligned}
$$

We notice that the second row is just the partial derivative with respect to $x$ of the first one. Hence, we just have to verify that expressions (4.3) and (4.4) satisfy the equation

$$
\phi_{1, r, n+1, t_{r}}=-\frac{f_{r, x}\left(u_{r, n+1}\right)}{2} \phi_{1, r, n+1}+f_{r}\left(u_{r, n+1}\right) \phi_{1, r, n+1, x} .
$$

Applying expression (4.4) and the induction hypothesis we obtain for the left hand side of this equation

$$
\phi_{1, r, n+1, t_{r}}=\frac{1}{2 n+1}\left(\phi_{1, r, n} \frac{\phi_{2, r, n, x}}{\phi_{2, r, n}}-\phi_{1, r, n, x}\right)\left(\frac{f_{r, x}\left(u_{r, n}\right)}{2}-f_{r}\left(u_{r, n}\right) \frac{\phi_{2, r, n, x}}{\phi_{2, r, n}}\right),
$$

and for the right hand side

$$
\begin{aligned}
& -\frac{f_{r, x}\left(u_{r, n+1}\right)}{2} \phi_{1, r, n+1}+f_{r}\left(u_{r, n+1}\right) \phi_{1, r, n+1, x} \\
& \quad=\frac{1}{2 n+1}\left(\phi_{1, r, n} \frac{\phi_{2, r, n, x}}{\phi_{2, r, n}}-\phi_{1, r, n, x}\right) \cdot\left(-\frac{f_{r, x}\left(u_{r, n+1}\right)}{2}-f_{r}\left(u_{r, n+1}\right) \frac{\phi_{2, r, n, x}}{\phi_{2, r, n}}\right) .
\end{aligned}
$$

Now, we prove that both expressions are equal. By applying the statement 2 of Theorem 3.1 for $\sigma=\frac{\phi_{2, r, n, x}}{\phi_{2, r, n}}$, expression (4.7) turns into

$$
\begin{aligned}
& -\frac{f_{r, x}\left(u_{r, n+1}\right)}{2}-f_{r}\left(u_{r, n+1}\right) \frac{\phi_{2, r, n, x}}{\phi_{2, r, n}}=-\frac{f_{r, x}\left(u_{r, n}\right)+A_{r, x}}{2}-\left(f_{r}\left(u_{r, n}\right)+A_{r}\right) \frac{\phi_{2, r, n, x}}{\phi_{2, r, n}} \\
& =-\frac{f_{r, x}\left(u_{r, n}\right)}{2}-f_{r}\left(u_{r, n}\right) \frac{\phi_{2, r, n, x}}{\phi_{2, r, n}}-\frac{A_{r, x}}{2}-A_{r} \frac{\phi_{2, r, n, x}}{\phi_{2, r, n}} \\
& =-\frac{f_{r, x}\left(u_{r, n}\right)}{2}-f_{r}\left(u_{r, n}\right) \frac{\phi_{2, r, n, x}}{\phi_{2, r, n}}-\frac{A_{r, x}}{2}+f_{r, x}\left(u_{r, n}\right)+\frac{A_{r, x}}{2} \\
& =\frac{f_{r, x}\left(u_{r, n}\right)}{2}-f_{r}\left(u_{r, n}\right) \frac{\phi_{2, r, n, x}}{\phi_{2, r, n}}
\end{aligned}
$$

which is equal to expression (4.6). Therefore, both sides of expression (4.5) coincide. 
Now we proceed as in [1]. We take another column solution $\left(\phi_{2, r, n+1}, \phi_{2, r, n+1, x}\right)^{t}$ of this system for potential $u_{r, n+1}$ which is linearly independent of the one we have just computed, i.e., $\operatorname{det} \mathcal{B}_{n+1,0}^{(r)}$ is a nontrivial constant. We take $\phi_{2, r, n+1}$ such that

$$
\operatorname{det} \mathcal{B}_{n+1,0}^{(r)}=2(n+1)+1 .
$$

We notice that with this condition we have

$$
\operatorname{det} \mathcal{B}_{n+1,0}^{(r)}=\phi_{2, r, n+1, x} \frac{\theta_{r, n}}{\theta_{r, n+1}}-\phi_{2, r, n+1} \frac{\theta_{r, n, x} \theta_{r, n+1}-\theta_{r, n} \theta_{r, n+1, x}}{\theta_{r, n+1}^{2}}=2(n+1)+1,
$$

multiplying both sides by $\theta_{r, n+1}^{2}$ and using the recursion formula (2.9) we get

$$
\phi_{2, r, n+1, x} \theta_{r, n} \theta_{r, n+1}-\phi_{2, r, n+1}\left(\theta_{r, n, x} \theta_{r, n+1}-\theta_{r, n} \theta_{r, n+1, x}\right)=\theta_{r, n+2, x} \theta_{r, n}-\theta_{r, n+2} \theta_{r, n, x} .
$$

Setting $\phi_{2, r, n+1}=\frac{\alpha_{2, r, n+1}}{\theta_{r, n+1}}$ yields to

$$
\alpha_{2, r, n+1, x} \theta_{r, n}-\alpha_{2, r, n+1} \theta_{r, n, x}=\theta_{r, n+2, x} \theta_{r, n}-\theta_{r, n+2} \theta_{r, n, x}
$$

thus, $\alpha_{2, r, n+1}=\theta_{r, n+2}$ and $\phi_{2, r, n+1}=\frac{\theta_{r, n+2}}{\theta_{r, n+1}}$. This concludes the proof.

Adler and Moser proved in [1] that matrix $\mathcal{B}_{n, 0}^{(r)}$ is a fundamental matrix for the Schrödinger equation (2.6) for $E=0$. But they did not prove there that this matrix is also a fundamental matrix for the second equation of the system (4.2). To do that, it is necessary to control the action of the Darboux transformations over the differential polynomials $f_{j}$, as we did in Section 3 .

Remark 4.2. Since $\phi_{1, r, n}=\frac{\theta_{r, n-1}}{\theta_{r, n}}$ and $\phi_{2, r, n}=\frac{\theta_{r, n+1}}{\theta_{r, n}}$ are solutions of Schrödinger equation (2.6) for $E=0$, this translate into the following equation for polynomials $\theta_{r, n}$ :

$$
\theta_{r, n+1, x x} \theta_{r, n}+\theta_{r, n+1} \theta_{r, n, x x}-2 \theta_{r, n, x} \theta_{r, n+1, x}=0 .
$$

Theorem 4.3. We have that

$$
\operatorname{det} \mathcal{B}_{n, 0}^{(r)}=2 n+1
$$

Example 4.4. To illustrate the results, we present explicit computations using SAGE of fundamental solutions of the system for the first values of $n$.

1. First, we show the first examples of unadjusted fundamental solutions

$$
\begin{array}{cccc}
n & \phi_{1, r, n} & \phi_{2, r, n} & u_{r, n} \\
0 & 1 & x & 0 \\
1 & \frac{1}{x} & \frac{x^{3}+\tau_{2}}{x} & \frac{2}{x^{2}} \\
2 & \frac{x}{x^{3}+\tau_{2}} & \frac{x^{6}+5 x^{3} \tau_{2}+x \tau_{3}-5 \tau_{2}^{2}}{x^{3}+\tau_{2}} & \frac{6 x\left(x^{3}-2 \tau_{2}\right)}{\left(x^{3}+\tau_{2}\right)^{2}} \\
3 & \frac{x^{3}+\tau_{2}}{x^{6}+5 x^{3} \tau_{2}+x \tau_{3}-5 \tau_{2}^{2}} & \frac{p_{1}\left(x, \tau_{2}, \tau_{3}, \tau_{4}\right)}{x^{6}+5 x^{3} \tau_{2}+x \tau_{3}-5 \tau_{2}^{2}} & \frac{p_{2}\left(x, \tau_{2}, \tau_{3}\right)}{\left(x^{6}+5 x^{3} \tau_{2}+x \tau_{3}-5 \tau_{2}^{2}\right)^{2}}
\end{array}
$$

where

$$
\begin{aligned}
& p_{1}\left(x, \tau_{2}, \tau_{3}, \tau_{4}\right)=x^{10}+15 x^{7} \tau_{2}+7 x^{5} \tau_{3}-35 x^{2} \tau_{2} \tau_{3}+175 x \tau_{2}^{3}-\frac{7}{3} \tau_{3}^{2}+x^{3} \tau_{4}+\tau_{2} \tau_{4}, \\
& p_{2}\left(x, \tau_{2}, \tau_{3}\right)=12 x^{10}-36 x^{5} \tau_{3}+450 x^{4} \tau_{2}^{2}+300 x \tau_{2}^{3}+2 \tau_{3}^{2} .
\end{aligned}
$$


2. Next, we compute fundamental solutions for potentials which are solutions of the first level of the KdV hierarchy, $\mathrm{KdV}_{1}$ equation: $u_{t_{1}}=\frac{3}{2} u u_{x}-\frac{1}{4} u_{x x x}$. We also show the explicit choice of the functions $\tau_{i}$

$$
\begin{array}{ccccc}
n & \phi_{1,1, n} & \phi_{2,1, n} & u_{1, n} & \left(\tau_{2}, \ldots, \tau_{n}\right) \\
0 & 1 & x & 0 & \left(3 t_{1}\right) \\
1 & \frac{1}{x} & \frac{x^{3}+3 t_{1}}{x} & \frac{2}{x^{2}} & \left(3 t_{1}, 0\right) \\
2 & \frac{x}{x^{3}+3 t_{1}} & \frac{x^{6}+15 x^{3} t_{1}-45 t_{1}^{2}}{x^{3}+3 t_{1}} & \frac{6 x\left(x^{3}-6 t_{1}\right)}{\left(x^{3}+3 t_{1}\right)^{2}} & \\
3 & \frac{x^{3}+3 t_{1}}{x^{6}+15 x^{3} t_{1}-45 t_{1}^{2}} & \frac{x^{10}+45 x^{7} t_{1}+4725 x t_{1}^{3}}{x^{6}+15 x^{3} t_{1}-45 t_{1}^{2}} & \frac{6 x\left(2 x^{9}+675 x^{3} t_{1}^{2}+1350 t_{1}^{3}\right)}{\left(x^{6}+15 x^{3} t_{1}-45 t_{1}^{2}\right)^{2}} & \left(3 t_{1}, 0,0\right)
\end{array}
$$

\subsection{Fundamental matrices for $E \neq 0$}

In this section, we compute explicitly fundamental matrices of system (2.2) when $u=u_{r, n}=$ $-2\left(\log \theta_{r, n}\right)_{x x}$ and $E \neq 0$. In this case, the system is

$$
\begin{aligned}
& \Phi_{x}=U \Phi=\left(\begin{array}{cr}
0 & 1 \\
u_{r, n}-E & 0
\end{array}\right) \Phi, \\
& \Phi_{t_{r}}=V_{r} \Phi=\left(\begin{array}{cc}
-\frac{F_{r, x}\left(u_{r, n}\right)}{2} & F_{r}\left(u_{r, n}\right) \\
\left(u_{r, n}-E\right) F_{r}\left(u_{r, n}\right)-\frac{F_{r, x x}\left(u_{r, n}\right)}{2} & \frac{F_{r, x}\left(u_{r, n}\right)}{2}
\end{array}\right) \Phi .
\end{aligned}
$$

The zero curvature condition of this system is still the $\mathrm{KdV}_{r}$ equation for $c_{i}=0, i=1, \ldots, r$ :

$$
u_{r, n, t_{r}}=2 f_{r+1, x}\left(u_{r, n}\right) .
$$

When $E \neq 0$, we take $\lambda \in \mathbb{C}$ a parameter over $K$ such that $E+\lambda^{2}=0$.

Next, we consider the differential systems

$$
\begin{aligned}
Q_{n, x x}^{+}= & Q_{n, x}^{+}\left(-2 \lambda+2 \frac{\theta_{r, n, x}}{\theta_{r, n}}\right)+Q_{n}^{+}\left(2 \lambda \frac{\theta_{r, n, x}}{\theta_{r, n}}-\frac{\theta_{r, n, x x}}{\theta_{r, n}}\right), \\
Q_{n, t_{r}}^{+}= & Q_{n, x}^{+} F_{r}\left(u_{r, n}\right) \\
& +Q_{n}^{+}\left(-(-1)^{r} \lambda^{2 r+1}+\lambda F_{r}\left(u_{r, n}\right)+\frac{\theta_{r, n, t_{r}}}{\theta_{r, n}}-\frac{F_{r, x}\left(u_{r, n}\right)}{2}-F_{r}\left(u_{r, n}\right) \frac{\theta_{r, n, x}}{\theta_{r, n}}\right), \\
Q_{n, x x}^{-}= & Q_{n, x}^{-}\left(2 \lambda+2 \frac{\theta_{r, n, x}}{\theta_{r, n}}\right)-Q_{n}^{-}\left(2 \lambda \frac{\theta_{r, n, x}}{\theta_{r, n}}+\frac{\theta_{r, n, x x}}{\theta_{r, n}}\right), \\
Q_{n, t_{r}}^{-}= & Q_{n, x}^{-} F_{r}\left(u_{r, n}\right) \\
& +Q_{n}^{-}\left((-1)^{r} \lambda^{2 r+1}-\lambda F_{r}\left(u_{r, n}\right)+\frac{\theta_{r, n, t_{r}}}{\theta_{r, n}}-\frac{F_{r, x}\left(u_{r, n}\right)}{2}-F_{r}\left(u_{r, n}\right) \frac{\theta_{r, n, x}}{\theta_{r, n}}\right) .
\end{aligned}
$$

We have the following relations for the solutions of the differential systems (4.10)-(4.11) and (4.12)-(4.13).

Lemma 4.5. Functions $Q_{n}^{+}$and $Q_{n}^{-}$recursively defined by

$$
Q_{0}^{+}=1, \quad Q_{n+1}^{+}=\frac{\lambda Q_{n}^{+} \theta_{r, n+1}+Q_{n, x}^{+} \theta_{r, n+1}-Q_{n}^{+} \theta_{r, n+1, x}}{\theta_{r, n}}
$$




$$
Q_{0}^{-}=1, \quad Q_{n+1}^{-}=\frac{\lambda Q_{n}^{-} \theta_{r, n+1}-Q_{n, x}^{-} \theta_{r, n+1}+Q_{n}^{-} \theta_{r, n+1, x}}{\theta_{r, n}}
$$

are solutions of the differential systems (4.10)-(4.11) and (4.12)-(4.13).

Proof. We prove it by induction on $n$. For $n=0$ we have $\theta_{r, 0}=1$, hence, $u_{r, 0}=0$ and $F_{r}\left(u_{r, 0}\right)=(-1)^{r} \lambda^{2 r}$. So, $Q_{0}^{+}=1$ and $Q_{0}^{-}=1$ are solutions of the systems $(4.10)-(4.11)$ and $(4.12)-(4.13)$.

Now, we suppose it is true for $n$ and prove it for $n+1$. We have to prove that expressions

$$
\begin{aligned}
Q_{n+1}^{+} & =\frac{\lambda Q_{n}^{+} \theta_{r, n+1}+Q_{n, x}^{+} \theta_{r, n+1}-Q_{n}^{+} \theta_{r, n+1, x}}{\theta_{r, n}}, \\
Q_{n+1}^{-} & =\frac{\lambda Q_{n}^{-} \theta_{r, n+1}-Q_{n, x}^{-} \theta_{r, n+1}+Q_{n}^{-} \theta_{r, n+1, x}}{\theta_{r, n}}
\end{aligned}
$$

satisfy equations (4.10), (4.11), (4.12) and (4.13) respectively, for $n+1$. First, we prove that $Q_{n+1}^{+}$satisfies (4.10) and (4.11). By induction hypothesis, we know that $Q_{n}^{+}$satisfies (4.10), using this expression and (4.8) we have

$$
\begin{aligned}
Q_{n+1, x}^{+} & =\frac{\lambda Q_{n}^{+} \theta_{r, n+1, x}-\lambda Q_{n, x}^{+} \theta_{r, n+1}}{\theta_{r, n}}+\frac{\left(\lambda Q_{n}^{+} \theta_{r, n+1}+Q_{n, x}^{+} \theta_{r, n+1}-Q_{n}^{+} \theta_{r, n+1, x}\right) \theta_{r, n, x}}{\theta_{r, n}^{2}}, \\
Q_{n+1, x x}^{+} & =\frac{Q_{n, x}^{+}}{\theta_{r, n}^{3}} p_{1}\left(x, t_{r}, \lambda\right)+\frac{Q_{n}^{+}}{\theta_{r, n}^{3}} p_{2}\left(x, t_{r}, \lambda\right),
\end{aligned}
$$

and

$$
\begin{aligned}
Q_{n+1, x}^{+} & \left(-2 \lambda+2 \frac{\theta_{r, n+1, x}}{\theta_{r, n+1}}\right)+Q_{n+1}^{+}\left(2 \lambda \frac{\theta_{r, n+1, x}}{\theta_{r, n+1}}-\frac{\theta_{r, n+1, x x}}{\theta_{r, n+1}}\right) \\
= & \frac{Q_{n, x}^{+}}{\theta_{r, n}^{3}} p_{1}\left(x, t_{r}, \lambda\right)+\frac{Q_{n}^{+}}{\theta_{r, n}^{3}} p_{2}\left(x, t_{r}, \lambda\right),
\end{aligned}
$$

where

$$
\begin{aligned}
& p_{1}\left(x, t_{r}, \lambda\right)=2 \lambda^{2} \theta_{r, n}^{2} \theta_{r, n+1}-2 \lambda \theta_{r, n} \theta_{r, n, x} \theta_{r, n+1}+2 \theta_{r, n} \theta_{r, n, x} \theta_{r, n+1, x}-\theta_{r, n}^{2} \theta_{r, n+1, x x}, \\
& p_{2}\left(x, t_{r}, \lambda\right)=-2 \lambda^{2} \theta_{r, n} \theta_{r, n, x} \theta_{r, n+1}+2 \lambda \theta_{r, n} \theta_{r, n, x x} \theta_{r, n+1}+\theta_{r, n}^{2} \theta_{r, n+1, x x}-\theta_{r, n} \theta_{r, n, x x} \theta_{r, n+1, x} .
\end{aligned}
$$

Thus, both expressions coincide and $Q_{n+1}^{+}$is solution of equation (4.10).

On the other hand, by induction hypothesis, we know that $Q_{n}^{+}$satisfies (4.11). Using this equation, expressions

$$
\begin{aligned}
\sigma_{2, r, n}= & \left(\log \phi_{2, r, n}\right)_{x}=\frac{\theta_{r, n+1, x} \theta_{r, n}-\theta_{r, n+1} \theta_{r, n, x}}{\theta_{r, n} \theta_{r, n+1}} \\
\sigma_{2, r, n, t_{r}}= & \frac{\theta_{r, n+1, x t_{r}}}{\theta_{r, n+1}}-\frac{\theta_{r, n, x t_{r}}}{\theta_{r, n}}+\frac{\theta_{r, n, x} \theta_{r, n, t_{r}}}{\theta_{r, n}^{2}}-\frac{\theta_{r, n+1, x} \theta_{r, n+1, t_{r}}}{\theta_{r, n+1}^{2}}, \\
Q_{n, x t_{r}}^{+}= & Q_{n, x}^{+}\left(-(-1)^{r} \lambda^{2 r+1}-\lambda F_{r}\left(u_{r, n}\right)+\frac{F_{r, x}\left(u_{r, n}\right)}{2}+F_{r}\left(u_{r, n}\right) \frac{\theta_{r, n, x}}{\theta_{r, n}}+\frac{\theta_{r, n, t_{r}}}{\theta_{r, n}}\right) \\
& +Q_{n}^{+}\left(2 \lambda F_{r}\left(u_{r, n}\right) \frac{\theta_{r, n, x}}{\theta_{r, n}}+\lambda F_{r, x}\left(u_{r, n}\right)-2 F_{r}\left(u_{r, n}\right) \frac{\theta_{r, n, x x}}{\theta_{r, n}}+F_{r}\left(u_{r, n}\right) \frac{\theta_{r, n, x}^{2}}{\theta_{r, n}^{2}}\right. \\
& \left.-\frac{F_{r, x x}\left(u_{r, n}\right)}{2}-F_{r, x}\left(u_{r, n}\right) \frac{\theta_{r, n, x}}{\theta_{r, n}}-\frac{\theta_{r, n, x} \theta_{r, n, t_{r}}}{\theta_{r, n}^{2}}+\frac{\theta_{r, n, x t_{r}}}{\theta_{r, n}}\right),
\end{aligned}
$$


the derivative with respect to $x$ of statement 2 of Corollary 3.4 and expression (3.6) for $\sigma_{2, r, n, t_{r}}$, we obtain

$$
Q_{n+1, t_{r}}^{+}=Q_{n, x}^{+} \frac{p_{3}\left(x, t_{r}, \lambda\right)}{\theta_{r, n}^{2}}+Q_{n}^{+} \frac{p_{4}\left(x, t_{r}, \lambda\right)}{\theta_{r, n}^{2}},
$$

where

$$
\begin{aligned}
p_{3}\left(x, t_{r}, \lambda\right)= & -(-1)^{r} \lambda^{2 r+1} \theta_{r, n} \theta_{r, n+1}+F_{r}\left(u_{r, n}\right) \theta_{r, n, x} \theta_{r, n+1}-F_{r}\left(u_{r, n}\right) \theta_{r, n} \theta_{r, n+1, x} \\
& +F_{r, x}\left(u_{r, n}\right) \frac{\theta_{r, n} \theta_{r, n+1}}{2}+\theta_{r, n} \theta_{r, n+1, t_{r}}, \\
p_{4}\left(x, t_{r}, \lambda\right)= & -(-1)^{r} \lambda^{2 r+2} \theta_{r, n} \theta_{r, n+1}+(-1)^{r} \lambda^{2 r+1} \theta_{r, n} \theta_{r, n+1, x}+\lambda^{2} F_{r}\left(u_{r, n}\right) \theta_{r, n} \theta_{r, n+1} \\
& +\lambda^{2} P_{r} \theta_{r, n} \theta_{r, n+1}+\lambda \theta_{r, n} \theta_{r, n+1, t_{r}}+\lambda F_{r}\left(u_{r, n}\right) \theta_{r, n, x} \theta_{r, n+1} \\
& +\lambda F_{r, x}\left(u_{r, n}\right) \frac{\theta_{r, n} \theta_{r, n+1}}{2}-\lambda F_{r}\left(u_{r, n}\right) \theta_{r, n} \theta_{r, n+1, x}+F_{r, x}\left(u_{r, n}\right) \frac{\theta_{r, n} \theta_{r, n+1, x}}{2} \\
& -P_{r} \theta_{r, n, x} \theta_{r, n+1, x}-\frac{\theta_{r, n} \theta_{r, n+1, x} \theta_{r, n+1, t_{r}}}{\theta_{r, n+1}}-F_{r}\left(u_{r, n}\right) \theta_{r, n, x} \theta_{r, n+1, x} \\
& +F_{r}\left(u_{r, n}\right) \frac{\theta_{r, n} \theta_{r, n+1, x}^{2}}{\theta_{r, n+1}}+P_{r} \frac{\theta_{r, n} \theta_{r, n+1, x}^{2}}{\theta_{r, n+1}}+P_{r, x} \frac{\theta_{r, n} \theta_{r, n+1, x}}{2} .
\end{aligned}
$$

Finally, using relation (4.10) for $Q_{n}^{+}$and statements 1 and 2 of Corollary 3.4, the right hand side of equation (4.11) for $Q_{n+1}^{+}$reads

$$
\begin{aligned}
& Q_{n+1, x}^{+} F_{r}\left(u_{r, n+1}\right)+Q_{n+1}^{+}\left(\lambda^{3}+\lambda F_{r}\left(u_{r, n+1}\right)+\frac{\theta_{r, n, t_{r}}}{\theta_{r, n}}-\frac{F_{r, x}\left(u_{r, n+1}\right)}{2}-F_{r}\left(u_{r, n+1}\right) \frac{\theta_{r, n, x}}{\theta_{r, n}}\right) \\
& \quad=Q_{n, x}^{+} \frac{p_{3}\left(x, t_{r}, \lambda\right)}{\theta_{r, n}^{2}}+Q_{n}^{+} \frac{p_{4}\left(x, t_{r}, \lambda\right)}{\theta_{r, n}^{2}} .
\end{aligned}
$$

Therefore, both expressions coincide and $Q_{n+1}^{+}$is a solution of equation (4.11).

The proof for $Q_{n+1}^{-}$is analogous.

As a consequence, we have the following result:

Theorem 4.6. Let $n$ be a non negative integer, then, for $E=-\lambda^{2} \neq 0$ and $u=u_{r, n}$, a fundamental matrix for system (4.9) is

$$
\mathcal{B}_{n, \lambda}^{(r)}=\left(\begin{array}{cc}
\phi_{r, n}^{+} & \phi_{r, n}^{-} \\
\phi_{r, n, x}^{+} & \phi_{r, n, x}^{-}
\end{array}\right)
$$

where

$$
\begin{aligned}
& \phi_{r, n}^{+}\left(x, t_{r}, \lambda\right)=e^{\lambda x+(-1)^{r} \lambda^{2 r+1} t_{r}} \frac{Q_{r, n}^{+}\left(x, t_{r}, \lambda\right)}{\theta_{r, n}}, \\
& \phi_{r, n}^{-}\left(x, t_{r}, \lambda\right)=e^{-\lambda x-(-1)^{r} \lambda^{2 r+1} t_{r}} \frac{Q_{r, n}^{-}\left(x, t_{r}, \lambda\right)}{\theta_{r, n}},
\end{aligned}
$$

where $Q_{r, n}^{+}$and $Q_{r, n}^{-}$are functions in $x, t_{r}, \lambda$ defined by means of Lemma 4.5.

Proof. We prove it by induction on $n$. For $n=0$ the definition $\theta_{r, 0}=1$ leads to $u_{r, 0}=0$. So, the system (4.9) becomes

$$
\left(\begin{array}{cc}
\phi_{r, 0, x}^{+} & \phi_{r, 0, x}^{-} \\
\phi_{r, 0, x x}^{+} & \phi_{r, 0, x x}^{-}
\end{array}\right)=\left(\begin{array}{cc}
0 & 1 \\
\lambda^{2} & 0
\end{array}\right)\left(\begin{array}{cc}
\phi_{r, 0}^{+} & \phi_{r, 0}^{-} \\
\phi_{r, 0, x}^{+} & \phi_{r, 0, x}^{-}
\end{array}\right),
$$




$$
\left(\begin{array}{cc}
\phi_{r, 0, t_{r}}^{+} & \phi_{r, 0, t_{r}}^{-} \\
\phi_{r, 0, x t_{r}}^{+} & \phi_{r, 0, x t_{r}}^{-}
\end{array}\right)=\left(\begin{array}{cc}
0 & (-1)^{r} \lambda^{2 r} \\
(-1)^{r} \lambda^{2 r+2} & 0
\end{array}\right)\left(\begin{array}{cc}
\phi_{r, 0}^{+} & \phi_{r, 0}^{-} \\
\phi_{r, 0, x}^{+} & \phi_{r, 0, x}^{-}
\end{array}\right)
$$

Hence, $\phi_{r, 0}^{+}=e^{\lambda x+(-1)^{r} \lambda^{2 r+1} t_{r}}$ and $\phi_{r, 0}^{-}=e^{-\lambda x-(-1)^{r} \lambda^{2 r+1} t_{r}}$ generate $\mathcal{B}_{0, \lambda}^{(r)}$. Since $\theta_{r, 0}=1$, we find $Q_{r, 0}^{ \pm}=1$, as in Lemma 4.5 .

Next, we suppose it true for $n$ and prove it for $n+1$. Since

$$
\phi_{r, n}^{+}\left(x, t_{r}, \lambda\right)=e^{\lambda x+(-1)^{r} \lambda^{2 r+1} t_{r}} \frac{Q_{r, n}^{+}}{\theta_{r, n}}, \quad \phi_{r, n}^{-}\left(x, t_{r}, \lambda\right)=e^{-\lambda x-(-1)^{r} \lambda^{2 r+1} t_{r}} \frac{Q_{r, n}^{-}}{\theta_{r, n}}
$$

are solutions of Schrödinger equation $\phi_{x x}=\left(u_{r, n}+\lambda^{2}\right) \phi$, we apply a Darboux transformation with $\phi_{2, r, n}=\frac{\theta_{r, n+1}}{\theta_{r, n}}$ to this equation and we obtain

$$
\begin{aligned}
\operatorname{DT}\left(\phi_{2, r, n}\right) u_{r, n} & =u_{r, n}-2\left(\log \phi_{2, r, n}\right)_{x x}=u_{r, n}-2 \sigma_{2, r, n, x}=u_{r, n+1}, \\
\operatorname{DT}\left(\phi_{2, r, n}\right) \phi_{r, n}^{+} & =\phi_{r, n, x}^{+}-\frac{\phi_{2, r, n, x}}{\phi_{2, r, n}} \phi_{r, n}^{+} \\
& =\frac{e^{\lambda x+(-1)^{r} \lambda^{2 r+1} t_{r}}}{\theta_{r, n+1}} \cdot \frac{\lambda Q_{r, n}^{+} \theta_{r, n+1}+Q_{r, n, x}^{+} \theta_{r, n+1}-Q_{r, n}^{+} \theta_{r, n+1, x}}{\theta_{r, n}} \\
& =e^{\lambda x+(-1)^{r} \lambda^{2 r+1} t_{r}} \frac{Q_{r, n+1}^{+}}{\theta_{r, n+1}}=\phi_{r, n+1}^{+}\left(x, t_{r}, \lambda\right), \\
\operatorname{DT}\left(\phi_{2, r, n}\right) \phi_{r, n}^{-} & =\phi_{r, n, x}^{-}-\frac{\phi_{2, r, n, x}}{\phi_{2, r, n}} \phi_{r, n}^{-} \\
& =\frac{e^{-\lambda x-(-1)^{r} \lambda^{2 r+1} t_{r}}}{\theta_{r, n+1}} \cdot \frac{-\lambda Q_{r, n}^{-} \theta_{r, n+1}+Q_{r, n, x}^{-} \theta_{r, n+1}-Q_{r, n}^{-} \theta_{r, n+1, x}}{\theta_{r, n}} \\
& =e^{-\lambda x-(-1)^{r} \lambda^{2 r+1} t_{r}} \frac{\left(-Q_{r, n+1}^{-}\right)}{\theta_{r, n+1}}=-\phi_{r, n+1}^{-}\left(x, t_{r}, \lambda\right),
\end{aligned}
$$

by Lemma 4.5. Hence, $\operatorname{DT}\left(\phi_{2, r, n}\right) \phi_{r, n}^{+}=\phi_{r, n+1}^{+}\left(x, t_{r}, \lambda\right)$ and $\operatorname{DT}\left(\phi_{2, r, n}\right) \phi_{r, n}^{-}=-\phi_{r, n+1}^{-}\left(x, t_{r}, \lambda\right)$ generate $\mathcal{B}_{n+1, \lambda}^{(r)}$. This ends the proof.

As far as we know, a general expression for fundamental matrices for system (4.9) has never been computed when $E \neq 0$. In the stationary case, i.e., in the case we only have the Schrödinger equation with Adler-Moser potentials, P. Clarkson showed in [9] an expression for the fundamental solutions of this equation when $E \neq 0$. However these expressions are not explicit, so it is not convenient for studying the Galois groups.

As in Theorem 4.1, the key to compute these solutions is to control the action of the Darboux transformations over the differential polynomials $f_{j}$, as we showed in Section 3. In Section 5 we will give some examples of these fundamental solutions both in the general framework of unadjusted functions $\tau_{i}$ and in the particular case $r=1$, in the same line as in Example 4.4.

Proposition 4.7. The functions $Q_{r, n}^{+}, Q_{r, n}^{-}$and the solutions $\phi_{r, n}^{+}, \phi_{r, n}^{-}$defined in Theorem 4.6 satisfy the relations

$$
Q_{r, n}^{+}\left(x, t_{r},-\lambda\right)=(-1)^{n} Q_{r, n}^{-}\left(x, t_{r}, \lambda\right) \quad \text { and } \quad \phi_{r, n}^{+}\left(x, t_{r},-\lambda\right)=(-1)^{n} \phi_{r, n}^{-}\left(x, t_{r}, \lambda\right) .
$$

Proof. We notice that

$$
\phi_{r, n}^{+}\left(x, t_{r},-\lambda\right)=e^{-\lambda x-(-1)^{r} \lambda^{2 r+1} t_{r}} \frac{Q_{r, n}^{+}\left(x, t_{r},-\lambda\right)}{\theta_{r, n}},
$$


since $\theta_{r, n}$ does not depend on $\lambda$. So, both relations are equivalent and it suffices to prove that $Q_{r, n}^{+}\left(x, t_{r},-\lambda\right)=(-1)^{n} Q_{r, n}^{-}\left(x, t_{r}, \lambda\right)$. We prove it by induction on $n$. For $n=0$, we have that $Q_{r, 0}^{+}=1=Q_{r, 0}^{-}$. Hence, $Q_{r, 0}^{+}\left(x, t_{r},-\lambda\right)=(-1)^{0} Q_{r, 0}^{-}\left(x, t_{r}, \lambda\right)$.

Using the expresions (4.14) and (4.15), we obtain

$$
\begin{aligned}
Q_{r, n+1}^{+}\left(x, t_{r},-\lambda\right) & =\frac{\left(-\lambda \theta_{r, n+1}-\theta_{r, n+1, x}\right) Q_{r, n}^{+}\left(x, t_{r},-\lambda\right)+Q_{r, n, x}^{+}\left(x, t_{r},-\lambda\right) \theta_{r, n+1}}{\theta_{r, n}} \\
& =\frac{(-1)^{n}\left(\left(-\lambda \theta_{r, n+1}-\theta_{r, n+1, x}\right) Q_{r, n}^{-}\left(x, t_{r}, \lambda\right)+Q_{r, n, x}^{-}\left(x, t_{r}, \lambda\right) \theta_{r, n+1}\right)}{\theta_{r, n}} \\
& =\frac{(-1)^{n+1}\left(\left(\lambda \theta_{r, n+1}+\theta_{r, n+1, x}\right) Q_{r, n}^{-}\left(x, t_{r}, \lambda\right)-Q_{r, n, x}^{-}\left(x, t_{r}, \lambda\right) \theta_{r, n+1}\right)}{\theta_{r, n}} \\
& =(-1)^{n+1} Q_{r, n+1}^{-}\left(x, t_{r}, \lambda\right),
\end{aligned}
$$

as we wanted to prove.

This corollary allows us to compute the determinant of $\mathcal{B}_{n, \lambda}^{(r)}$. First observe that

$$
\begin{aligned}
\operatorname{det} \mathcal{B}_{n, \lambda}^{(r)} & =W\left(\phi_{r, n}^{+}, \phi_{r, n}^{-}\right)=(-1)^{n} W\left(\phi_{r, n}^{+}\left(x, t_{r}, \lambda\right), \phi_{r, n}^{+}\left(x, t_{r},-\lambda\right)\right) \\
& =(-1)^{n+1} \frac{2 \lambda Q_{r, n}^{+}\left(x, t_{r}, \lambda\right) Q_{r, n}^{+}\left(x, t_{r},-\lambda\right)+W\left(Q_{r, n}^{+}\left(x, t_{r},-\lambda\right), Q_{r, n}^{+}\left(x, t_{r}, \lambda\right)\right)}{\theta_{r, n}^{2}}
\end{aligned}
$$

where $W\left(\phi_{1}, \phi_{2}\right)=\phi_{1} \phi_{2, x}-\phi_{1, x} \phi_{2}$ denotes the Wronskian of $\phi_{1}$ and $\phi_{2}$.

Theorem 4.8. We have

$$
\operatorname{det} \mathcal{B}_{n, \lambda}^{(r)}=-2 \lambda^{2 n+1} .
$$

Proof. We proceed by induction on $n$. For $n=0$ we obtain $Q_{r, 0}^{+}=1$ and $\theta_{r, 0}=1$, so $\operatorname{det} \mathcal{B}_{0, \lambda}^{(r)}=$ $-2 \lambda$. Now, we suppose it is true for $n$ and prove it for $n+1$. Replacing expression (4.14) for $Q_{r, n+1}^{+}\left(x, t_{r}, \lambda\right)$ and $Q_{r, n+1}^{+}\left(x, t_{r},-\lambda\right)$ in formula (4.18) and using Proposition 4.7 and the induction hypothesis, we get

$$
\operatorname{det} \mathcal{B}_{n+1, \lambda}^{(r)}=-2 \lambda^{2 n+3}=-2 \lambda^{2(n+1)+1} .
$$

As we wanted to prove.

Remark 4.9. Theorem 4.8 implies that the matrix $\mathcal{B}_{n, \lambda}^{(r)}$ is not a fundamental matrix of system (2.2) for $\lambda=E=0$, since it is not invertible for that value of $E$. The reason of this is that, by Proposition 4.7 , when $\lambda=0$ we have $\phi_{r, n}^{+}\left(x, t_{r}, 0\right)=(-1)^{n} \phi_{r, n}^{-}\left(x, t_{r}, 0\right)$, so, both column solutions are linearly dependent. We will detail this phenomenon in Section 6 . In fact, we will show that it is not the same to set $E=0$ in (2.2) and then solve the system, than to solve the system for a generic $E$ and then replace $E=0$ in the solution obtained, i.e., there is not a specialization process in this sense.

Example 4.10. For $n=0$ and $n=1$ we obtain by direct computations the following solutions:

$$
\begin{array}{ccc}
n & \phi_{r, n}^{+} & \phi_{r, n}^{-} \\
0 & e^{\lambda x+(-1)^{r} \lambda^{2 r+1} t_{r}} & e^{-\lambda x-(-1)^{r} \lambda^{2 r+1} t_{r}} \\
1 & e^{\lambda x+(-1)^{r} \lambda^{2 r+1} t_{r} \frac{\lambda x-1}{x}} & e^{-\lambda x-(-1)^{r} \lambda^{2 r+1} t_{r}} \frac{\lambda x+1}{x}
\end{array}
$$


In next section we will show a method to compute functions $Q_{r, n}^{+}$and $Q_{r, n}^{-}$more efficient than solving explicitly equations (4.10), (4.11), (4.12) and (4.13). This allow us to obtain fundamental matrices $\mathcal{B}_{n, \lambda}^{(r)}$. In particular $\phi_{r, 1}^{+}$and $\phi_{r, 1}^{-}$are linearly independent solutions for the Schrödinger operator $-\partial^{2}+u_{r, 1}-E=0$ where $u_{r, 1}=2 / x^{2}$ is the constructed rational $\mathrm{KdV}_{r}$ potential, as long as $E \neq 0$.

\section{Examples of fundamental matrices for the case $E \neq 0$}

Along this section we will prove that the funtions $Q_{r, n}^{ \pm}$defined in Theorem 4.6 satisfy the recursion formula (2.9). This implies in particular that they are polynomials of $x$ with coefficients in $\mathbb{C}\left(\lambda, t_{r}\right)$. Thus, they generalize the family of Adler-Moser polynomials $\theta_{n}$.

For the following computations we do not suppose that functions $\theta_{n}$ and $Q_{n}^{ \pm}$and potentials $u_{n}$ are adjusted to any level of the KdV hierarchy.

\subsection{Generalized Adler-Moser polynomials}

In Lemma 4.5 we have obtained the recursive formulas (4.14) and (4.15) for $Q_{r, n}^{ \pm}$. As we have seen in the proof of Theorem 4.6, these expressions are obtained by applying Darboux-Crum transformations with $\phi_{2, r, n}$ to $\phi_{r, n}^{+}$and $\phi_{r, n}^{-}$, see expressions (4.16) and (4.17). For our present discussion, we consider the unadjusted relations given in Lemma 4.5:

$$
\begin{aligned}
Q_{n+1}^{+} & =\frac{\lambda Q_{n}^{+} \theta_{n+1}+Q_{n, x}^{+} \theta_{n+1}-Q_{n}^{+} \theta_{n+1, x}}{\theta_{n}}, \\
Q_{n+1}^{-} & =\frac{\lambda Q_{n}^{-} \theta_{n+1}-Q_{n, x}^{-} \theta_{n+1}+Q_{n}^{-} \theta_{n+1, x}}{\theta_{n}} .
\end{aligned}
$$

If we proceed in the same way performing Darboux transformations with $\phi_{1, r, n}$ we obtain that functions

$$
\begin{aligned}
\operatorname{DT}\left(\phi_{1, r, n}\right) \phi_{r, n}^{+} & =\phi_{r, n, x}^{+}-\frac{\phi_{1, r, n, x}}{\phi_{1, r, n}} \phi_{r, n}^{+} \\
& =\frac{e^{\lambda x+(-1)^{r} \lambda^{2 r+1} t_{r}}}{\theta_{r, n-1}} \frac{\lambda Q_{r, n}^{+} \theta_{r, n-1}+Q_{r, n, x}^{+} \theta_{r, n-1}-\theta_{r, n-1, x} Q_{r, n}^{+}}{\theta_{r, n}}, \\
\operatorname{DT}\left(\phi_{1, r, n}\right) \phi_{r, n}^{-} & =\phi_{r, n, x}^{-}-\frac{\phi_{1, r, n, x}}{\phi_{1, r, n}} \phi_{r, n}^{-} \\
& =\frac{e^{-\lambda x-(-1)^{r} \lambda^{2 r+1} t_{r}}}{\theta_{r, n-1}} \frac{-\lambda Q_{r, n}^{-} \theta_{r, n-1}+Q_{r, n, x}^{-} \theta_{r, n-1}-\theta_{r, n-1, x} Q_{r, n}^{-}}{\theta_{r, n}},
\end{aligned}
$$

are solutions of Schrödinger equation for $E \neq 0$ and potential

$$
\operatorname{DT}\left(\phi_{1, r, n}\right) u_{r, n}=u_{r, n}-2\left(\log \phi_{1, r, n}\right)_{x x}=u_{r, n-1} .
$$

In the same way that we did for the functions (4.14) and (4.15), we can prove that the expressions

$$
\begin{aligned}
Q_{r, n-1}^{+} & :=\frac{\lambda Q_{r, n}^{+} \theta_{r, n-1}+Q_{r, n, x}^{+} \theta_{r, n-1}-\theta_{r, n-1, x} Q_{r, n}^{+}}{\lambda^{2} \theta_{r, n}}, \\
Q_{r, n-1}^{-} & :=\frac{\lambda Q_{r, n}^{-} \theta_{r, n-1}-Q_{r, n, x}^{-} \theta_{r, n-1}+\theta_{r, n-1, x} Q_{r, n}^{-}}{\lambda^{2} \theta_{r, n}}
\end{aligned}
$$

satisfy differential systems (4.10)-(4.11) and (4.12)-(4.13), respectively, for $n-1$. So, we obtain

$$
\mathrm{DT}\left(\phi_{1, r, n}\right) \phi_{r, n}^{+}=\phi_{r, n, x}^{+}-\frac{\phi_{1, r, n, x}}{\phi_{1, r, n}} \phi_{r, n}^{+}=\lambda^{2} \phi_{r, n-1}^{+},
$$




$$
\operatorname{DT}\left(\phi_{1, r, n}\right) \phi_{r, n}^{-}=\phi_{r, n, x}^{-}-\frac{\phi_{1, r, n, x}}{\phi_{1, r, n}} \phi_{r, n}^{-}=-\lambda^{2} \phi_{r, n-1}^{-} .
$$

For our present discussion, we just write

$$
\begin{aligned}
Q_{n-1}^{+} & =\frac{\lambda Q_{n}^{+} \theta_{n-1}+Q_{n, x}^{+} \theta_{n-1}-\theta_{n-1, x} Q_{n}^{+}}{\lambda^{2} \theta_{n}}, \\
Q_{n-1}^{-} & =\frac{\lambda Q_{n}^{-} \theta_{n-1}-Q_{n, x}^{-} \theta_{n-1}+\theta_{n-1, x} Q_{n}^{-}}{\lambda^{2} \theta_{n}} .
\end{aligned}
$$

Now, we can prove the following result:

Theorem 5.1. Functions $Q_{n}^{+}\left(x, t_{r}, \lambda\right)$ and $Q_{n}^{-}\left(x, t_{r}, \lambda\right)$ satisfy the differential recursions

$$
\begin{array}{lll}
Q_{0}^{+}=1, & Q_{1}^{+}=\lambda x-1, & Q_{n+1, x}^{+} Q_{n-1}^{+}-Q_{n+1}^{+} Q_{n-1, x}^{+}=(2 n+1) Q_{n}^{+2}, \\
Q_{0}^{-}=1, & Q_{1}^{-}=\lambda x+1, & Q_{n+1, x}^{-} Q_{n-1}^{-}-Q_{n+1}^{-} Q_{n-1, x}^{-}=(2 n+1) Q_{n}^{-2} .
\end{array}
$$

Proof. In Remark 4.10 we have computed $\phi_{n}^{+}$and $\phi_{n}^{-}$for $n=0$ and 1 . We have obtained $Q_{0}^{ \pm}=1, Q_{1}^{+}=\lambda x-1$ and $Q_{1}^{-}=\lambda x+1$. So, we just have to prove the recursion formulas. First, we prove (5.6). For this, we compute $Q_{n+1, x}^{+}$and $Q_{n-1, x}^{+}$using expressions (5.1) and (5.4):

$$
\begin{aligned}
Q_{n+1, x}^{+}= & \frac{1}{\theta_{n}^{2}}\left(\left(\lambda Q_{n, x}^{+} \theta_{n+1}+\lambda Q_{n}^{+} \theta_{n+1, x}+Q_{n, x x}^{+} \theta_{n+1}-Q_{n}^{+} \theta_{n+1, x x}\right) \theta_{n}\right. \\
& \left.+\left(Q_{n}^{+} \theta_{n+1, x}-\lambda Q_{n}^{+} \theta_{n+1}-Q_{n, x}^{+} \theta_{n+1}\right) \theta_{n, x}\right), \\
Q_{n-1, x}^{+}= & \frac{1}{\lambda^{2} \theta_{n}^{2}}\left(\left(\lambda Q_{n, x}^{+} \theta_{n-1}+\lambda Q_{n}^{+} \theta_{n-1, x}+Q_{n, x x}^{+} \theta_{n-1}-Q_{n}^{+} \theta_{n-1, x x}\right) \theta_{n}\right. \\
& \left.+\left(Q_{n}^{+} \theta_{n-1, x}-\lambda Q_{n}^{+} \theta_{n-1}-Q_{n, x}^{+} \theta_{n-1}\right) \theta_{n, x}\right) .
\end{aligned}
$$

Replacing this expressions in the recursion formula (5.6) we get

$$
\begin{aligned}
Q_{n+1, x}^{+} Q_{n-1}^{+}-Q_{n+1}^{+} Q_{n-1, x}^{+}= & \frac{\left(\lambda^{2} Q_{n}^{+}+2 \lambda Q_{n}^{+} Q_{n, x}^{+}+Q_{n}^{+} Q_{n, x x}^{+}\right)\left(\theta_{n+1, x} \theta_{n-1}-\theta_{n+1} \theta_{n-1, x}\right)}{\lambda^{2} \theta_{n}^{3}} \\
& +\frac{\left(\lambda Q_{n}^{+2}+Q_{n}^{+} Q_{n, x}^{+}\right)\left(\theta_{n+1} \theta_{n-1, x x}-\theta_{n+1, x x} \theta_{n-1}\right)}{\lambda^{2} \theta_{n}^{3}} \\
& +\frac{Q_{n}^{+2}\left(\theta_{n+1, x x} \theta_{n-1, x}-\theta_{n+1, x} \theta_{n-1, x x}\right)}{\lambda^{2} \theta_{n}^{2}} .
\end{aligned}
$$

We want to compute the expressions for $\theta_{n+1}$ and $\theta_{n-1}$ in brackets in terms of $\theta_{n}$. The first expression is just the relation (2.9). Now, if we derivate with respect to $x$ expression (2.9), we find the second one

$$
\theta_{n+1, x x} \theta_{n-1}-\theta_{n+1} \theta_{n-1, x x}=2(2 n+1) \theta_{n} \theta_{n, x} .
$$

In order to compute

$$
\theta_{n+1, x x} \theta_{n-1, x}-\theta_{n+1, x} \theta_{n-1, x x}
$$

we use relation (4.8). We have

$$
\theta_{n+1, x x}=2 \frac{\theta_{n+1, x} \theta_{n, x}}{\theta_{n}}-\frac{\theta_{n+1} \theta_{n, x x}}{\theta_{n}} \quad \text { and } \quad \theta_{n-1, x x}=2 \frac{\theta_{n-1, x} \theta_{n, x}}{\theta_{n}}-\frac{\theta_{n-1} \theta_{n, x x}}{\theta_{n}} .
$$

Replacing both expressions in (5.9) we get the third one

$$
\theta_{n+1, x x} \theta_{n-1, x}-\theta_{n+1, x} \theta_{n-1, x x}=\frac{\theta_{n, x x}}{\theta_{n}}\left(\theta_{n+1, x} \theta_{n-1}-\theta_{n+1} \theta_{n-1, x}\right)=(2 n+1) \theta_{n} \theta_{n, x x} .
$$


Applying the expressions (2.9), (5.8) and (5.10) we get

$$
\begin{aligned}
& Q_{n+1, x}^{+} Q_{n-1}^{+}-Q_{n+1}^{+} Q_{n-1, x}^{+}= \\
& =(2 n+1) \frac{\left(\lambda^{2} Q_{n}^{+2}+2 \lambda Q_{n}^{+} Q_{n, x}^{+}+Q_{n}^{+} Q_{n, x x}^{+}\right) \theta_{n}-2 \lambda Q_{n}^{+2} \theta_{n, x}-2 Q_{n}^{+} Q_{n, x}^{+} \theta_{n, x}+Q_{n}^{+2} \theta_{n, x x}}{\lambda^{2} \theta_{n}} .
\end{aligned}
$$

Finally, the expression (4.10) for $Q_{n, x x}^{+}$yields to

$$
Q_{n+1, x}^{+} Q_{n-1}^{+}-Q_{n+1}^{+} Q_{n-1, x}^{+}=(2 n+1) Q_{n}^{+2} .
$$

Analogously, the second recursion formula can be proved. So we have established our result.

Remark 5.2. By Lemmas 4.5 and 2.1 for $F=\mathbb{C}\left(\lambda, t_{r}\right)$ and $a=\lambda, b=-1$, we can conclude from this theorem that the functions $Q_{n}^{ \pm}\left(x, t_{r}, \lambda\right)$ are polynomials of $x$ and $\lambda$ with coefficients in $\mathbb{C}\left(t_{r}\right)$ for all $n$. Indeed, their degree as polynomials of $\lambda$ is $n$. Thus, Theorems 4.6 and 5.1 determine the algebraic structure of $\phi_{r, n}^{+}$and $\phi_{r, n}^{-}$.

Since polynomials $Q_{n}^{ \pm}$are not adjusted to any level of the KdV hierarchy, when we iterate the recurrences (5.6) and (5.7) we will obtain integration constants of $x$ which may depend on $\lambda$ and $\tau_{2}, \ldots, \tau_{n}$. We will denote such integration constants by $\tau_{2}^{ \pm}, \ldots, \tau_{n}^{ \pm}$.

Example 5.3. For the first polynomials we find

$\begin{array}{ccc}n & Q_{n}^{+} & Q_{n}^{-} \\ 0 & 1 & 1 \\ 1 & \lambda x-1 & \lambda x+1 \\ 2 & \lambda^{2} x^{3}-3 \lambda x^{2}+3 x+\tau_{2}^{+} & \lambda^{2} x^{3}+3 \lambda x^{2}+3 x+\tau_{2}^{-} \\ 3 & Q_{3}^{+} & Q_{3}^{-}\end{array}$

where

$$
\begin{aligned}
& Q_{3}^{+}=\lambda^{3} x^{6}-6 \lambda^{2} x^{5}+15 \lambda x^{4}-15 x^{3}+5 \lambda x^{3} \tau_{2}^{+}-15 x^{2} \tau_{2}^{+}-\left(\lambda \tau_{3}^{+}+5\left(\tau_{2}^{+}\right)^{2}\right) x+\tau_{3}^{+}, \\
& Q_{3}^{-}=\lambda^{3} x^{6}+6 \lambda^{2} x^{5}+15 \lambda x^{4}+15 x^{3}+5 \lambda x^{3} \tau_{2}^{-}+15 x^{2} \tau_{2}^{-}+\left(\lambda \tau_{3}^{-}+5\left(\tau_{2}^{-}\right)^{2}\right) x+\tau_{3}^{-} .
\end{aligned}
$$

\subsection{Examples of fundamental matrices for the case $E \neq 0$}

We can compute fundamental matrices for system (4.9) for any $n$ using recursion formulas (5.6) and (5.7).

Example 5.4. We present explicit computations using SAGE for the fundamental solutions of the system (4.9) when $E=-\lambda^{2} \neq 0$ for same potentials as in Example 4.4.

1. We first expose examples of unadjusted fundamental solutions:

$$
\begin{array}{ccc}
n & \phi_{r, n}^{+} & \phi_{r, n}^{-} \\
0 & e^{\lambda x+(-1)^{r} \lambda^{2 r+1} t_{r}} & e^{-\lambda x-(-1)^{r} \lambda^{2 r+1} t_{r}} \\
1 & e^{\lambda x+(-1)^{r} \lambda^{2 r+1} t_{r} \frac{\lambda x-1}{x}} & e^{-\lambda x-(-1)^{r} \lambda^{2 r+1} t_{r}} \frac{\lambda x+1}{x} \\
2 & e^{\lambda x+(-1)^{r} \lambda^{2 r+1} t_{r}} \frac{\lambda^{2} x^{3}-3 \lambda x^{2}+3 x+\tau_{2}^{+}}{x^{3}+\tau_{2}} & e^{-\lambda x-(-1)^{r} \lambda^{2 r+1} t_{r}} \frac{\lambda^{2} x^{3}+3 \lambda x^{2}+3 x+\tau_{2}^{-}}{x^{3}+\tau_{2}} \\
3 & e^{\lambda x+(-1)^{r} \lambda^{2 r+1} t_{r}} \frac{Q_{3}^{+}\left(\lambda, x, t_{r}\right)}{x^{6}+5 x^{3} \tau_{2}+x \tau_{3}-5 \tau_{2}^{2}} & e^{-\lambda x-(-1)^{r} \lambda^{2 r+1} t_{r}} \frac{Q_{3}^{-}\left(\lambda, x, t_{r}\right)}{x^{6}+5 x^{3} \tau_{2}+x \tau_{3}-5 \tau_{2}^{2}}
\end{array}
$$

where $Q_{3}^{+}$and $Q_{3}^{-}$are the ones given in (5.11). 
2. Next, we expose fundamental solutions for potentials which are solutions of the first level of the $\mathrm{KdV}$ hierarchy, $\mathrm{KdV}_{1}$ equation: $u_{t_{1}}=\frac{3}{2} u u_{x}-\frac{1}{4} u_{x x x}$. We also show the explicit choice of the functions $\tau_{i}^{ \pm}$. The choice of functions $\tau_{i}$ is the same as in Example 4.4:

$$
\begin{array}{cccc}
n & \phi_{1, n}^{+} & \phi_{1, n}^{-} & \left(\tau_{2}^{ \pm}, \ldots, \tau_{n}^{ \pm}\right) \\
0 & e^{\lambda x-\lambda^{3} t_{1}} & e^{-\lambda x+\lambda^{3} t_{1}} & \\
1 & e^{\lambda x-\lambda^{3} t_{1}} \frac{\lambda x-1}{x} & e^{-\lambda x+\lambda^{3} t_{1}} \frac{\lambda x+1}{x} & \\
2 & e^{\lambda x-\lambda^{3} t_{1}} \frac{\lambda^{2} x^{3}-3 \lambda x^{2}+3 x+3 \lambda^{2} t_{1}}{x^{3}+3 t_{1}} & e^{-\lambda x+\lambda^{3} t_{1}} \frac{\lambda^{2} x^{3}+3 \lambda x^{2}+3 x+3 \lambda^{2} t_{1}}{x^{3}+3 t_{1}} & \left(3 \lambda^{2} t_{1}\right) \\
3 & e^{\lambda x-\lambda^{3} t_{1}} \frac{Q_{3}^{+}\left(\lambda, x, t_{1}\right)}{x^{6}+15 x^{3} t_{1}-45 t_{1}^{2}} & e^{-\lambda x+\lambda^{3} t_{1}} \frac{Q_{3}^{-}\left(\lambda, x, t_{1}\right)}{x^{6}+15 x^{3} t_{1}-45 t_{1}^{2}} & \left(3 \lambda^{2} t_{1},-45\left(\lambda^{3} t_{1}^{2} \pm t_{1}\right)\right)
\end{array}
$$

where

$$
\begin{aligned}
Q_{3}^{+}\left(\lambda, x, t_{1}\right)= & \lambda^{3} x^{6}-6 \lambda^{2} x^{5}+15 \lambda x^{4}-15 x^{3}+15 \lambda^{3} x^{3} t_{1}-45 \lambda^{2} x^{2} t_{1}+45 \lambda x t_{1} \\
& -45 \lambda^{3} t_{1}^{2}-45 t_{1}, \\
Q_{3}^{-}\left(\lambda, x, t_{1}\right)= & \lambda^{3} x^{6}+6 \lambda^{2} x^{5}+15 \lambda x^{4}+15 x^{3}+15 \lambda^{3} x^{3} t_{1}+45 \lambda^{2} x^{2} t_{1}+45 \lambda x t_{1} \\
& -45 \lambda^{3} t_{1}^{2}+45 t_{1} .
\end{aligned}
$$

\section{Spectral curves and Darboux-Crum transformations}

Let $\Gamma_{n} \subset \mathbb{C}^{2}$ be the spectral curve associated to the stationary Schrödinger operator $-\partial_{x x}+u-E$ where $u$ is a s-KdV $\mathrm{K}_{n}$ potential. Next we consider the Zariski closure of $\Gamma_{n}$, say $\bar{\Gamma}_{n}$, in the complex projective plane $\mathbb{P}^{2}$. Let be

$$
p(E, \mu)=\mu^{2}-R_{2 n+1}(E)=\mu^{2}-\sum_{j=0}^{2 n+1} C_{j} E^{j}=0
$$

an equation for $\Gamma_{n}$. Then an equation for $\bar{\Gamma}_{n}$ is

$$
p_{h}(E, \mu, \nu)=\mu^{2} \nu^{2 n-1}-\widehat{R}_{2 n+1}(E, \nu)=0,
$$

where

$$
\widehat{R}_{2 n+1}(E, \nu)=\nu^{2 n+1} R_{2 n+1}\left(\frac{E}{\nu}\right)=\sum_{j=0}^{2 n+1} C_{j} \nu^{2 n+1-j} E^{j}
$$

is an homogeneous polynomial of degree $2 n+1$. Moreover, observe that the singular points of $\bar{\Gamma}_{n}$ are

$$
\operatorname{Sing}\left(\bar{\Gamma}_{n}\right)=\left\{(E, 0): E \text { is a multiple root of } R_{2 n+1}\right\} \cup\left\{P_{\infty}=[0: 1: 0]\right\} \text {, }
$$

and also

$$
\bar{\Gamma}_{n} \cap\{E=0\}=\left\{[0: \mu: \nu] \in \mathbb{P}^{2}: \mu^{2} \nu^{2 n-1}=C_{0} \nu^{2 n+1}\right\} .
$$




\subsection{Extended Green's function}

Following [15], we define the Green's function on $\Gamma_{n} \times \mathbb{C}$ as

$$
g(E, \mu, x)=\frac{\phi_{1} \phi_{2}}{W\left(\phi_{1}, \phi_{2}\right)},
$$

where $\phi_{1}$ and $\phi_{2}$ are two independent solutions of Schrödinger equation

$$
(L-E) \phi=\left(-\partial_{x x}+u-E\right) \phi=0 .
$$

for the same value of $E$ and $W\left(\phi_{1}, \phi_{2}\right)$ stands for their Wronskian.

Let

$$
\sigma_{+}=\sigma(E, \mu)=\frac{i \mu+F_{n, x} / 2}{F_{n}}, \quad \sigma_{-}=\sigma(E,-\mu)=\frac{-i \mu+F_{n, x} / 2}{F_{n}}
$$

be functions defined over the spectral curve. We recall the following result:

Lemma 6.1 ([15, Lemma 1.8]). Let $u$ be solution of $s-K d V_{n}$ equation (2.8). Let $\phi_{1}$ and $\phi_{2}$ be solutions of Schrödinger equation (6.2) for this potential and with corresponding functions over the spectral curve $\sigma_{+}$and $\sigma_{-}$defined by (6.3). Then $\sigma_{+}$and $\sigma_{-}$are solutions of the Riccati type equation

$$
\sigma^{2}+\sigma_{x}=u-E
$$

Moreover, the following identities are satisfied

$$
\begin{aligned}
& \sigma_{+}+\sigma_{-}=\frac{F_{n, x}}{F_{n}}=\frac{\left(\phi_{1} \phi_{2}\right)_{x}}{\phi_{1} \phi_{2}}, \quad \sigma_{+}-\sigma_{-}=\frac{2 i \mu}{F_{n}}=-\frac{W\left(\phi_{1}, \phi_{2}\right)}{\phi_{1} \phi_{2}}, \\
& \sigma_{+} \cdot \sigma_{-}=\frac{H_{n}}{F_{n}}=\frac{\phi_{1, x} \phi_{2, x}}{\phi_{1} \phi_{2}}
\end{aligned}
$$

where $W\left(\phi_{1}, \phi_{2}\right)=\phi_{1} \phi_{2, x}-\phi_{1, x} \phi_{2}$ denotes the Wronskian of $\phi_{1}$ and $\phi_{2}$.

We remark that this lemma is essentially a reformulation of a classic result that goes back to Hermite when he was studying closed form solutions for Lamé equation [17]. In [24] call this approach the Lindeman-Stieljes theory but, as far as we know, this approach was used for the first time by Hermite, and then by others: Halphen, Brioschi, Crawford, Stieljes, .... The method used that the product of solutions $X=\phi_{1} \phi_{2}$ is a solution of the second symmetric power of the Schrödinger equation

$$
\left(-\partial_{x x x}-4(u-E) \partial_{x}-2 u_{x}\right) X=0 .
$$

Then the relations (6.5) connect the solutions of the Riccati equation with that of the second symmetric power. The fact that there is a connection between the solutions of the second symmetric product and the Riccati equation of the Schrödinger equation is relevant for the differential Galois theory, although we will not use explicitely this connection in this paper. Furthermore it is interesting to point out that the solutions of the Lamé equation obtained by Hermite in [17], are associated to other algebro-geometric solutions of $\mathrm{KdV}$, finite-gap solutions

with regular spectral curves, see [21] and references therein. As far as we know, the relevance of the equation (6.6) for the KdV equation was considered for the first time by Gel'fand and Dikii in their fundamental paper about the asymptotic behaviour of the resolvent of the Schrödinger equation associated to the $\mathrm{KdV}$ equation [14]. 
By Lemma 6.1, the Green's function can be rewritten as

$$
g(E, \mu, x)=\frac{i F_{n}(E, x)}{2 \mu}=\frac{1}{\sigma_{-}-\sigma_{+}} .
$$

Observe that $g$ is well defined whenever $\mu \neq 0$, i.e., for energy levels such that $R_{2 n+1}(E) \neq 0$.

Next, let define a extension of $g$ on $\bar{\Gamma}_{n} \times \mathbb{C}_{x}$ as

$$
g_{h}(E, \mu, \nu, x)=\frac{i \nu^{n} F_{n}(E / \nu, x)}{2 \mu \nu^{n-1}}, \quad \text { for } \quad[E: \mu: \nu] \in \bar{\Gamma}_{n} \backslash\{\mu \nu=0\} .
$$

We call $g_{h}$ the homogenized Green's function. Next we will show that $g_{h}$ is well defined and also that it extends $g$, that is $g_{h}(E, \mu, 1, x)=g(E, \mu, x)$ for $(E, \mu, x) \in \Gamma_{n} \times \mathbb{C}_{x}$. To do that, observe that

$$
g_{h}(E, \mu, 1, x)=g(E, \mu, x) \quad \text { and } \quad g_{h}(a E, a \mu, a \nu, x)=g_{h}(E, \mu, \nu, x),
$$

for any $a \in \mathbb{C}, a \neq 0$. Moreover, we have that

$$
\widehat{F}_{n}(E, \nu, x):=\nu^{n} F_{n}(E / \nu, x)=\sum_{j=0}^{n} f_{n-j} \nu^{n-j} E^{j}
$$

is an homogeneous polynomial in $E$ of degree $n$ and then

$$
g_{h}(E, \mu, \nu, x)=\frac{i \widehat{F}_{n}(E, \nu, x)}{2 \mu \nu^{n-1}}, \quad \text { for } \quad[E: \mu: \nu] \in \bar{\Gamma}_{n}
$$

Also, we get the following formula

$$
\mu^{2} \nu^{2 n-2}=\nu^{2 n} R_{2 n+1}(E / \nu)=\frac{\nu \widehat{F}_{n} \widehat{F}_{n, x x}}{2}-(u-E / \nu) \widehat{F}_{n}^{2}-\frac{\nu^{2} \widehat{F}_{n, x}^{2}}{4},
$$

where

$$
\widehat{F}_{n, x}=\nu^{n-1} F_{n, x}(E / \nu) \quad \text { and } \quad \widehat{F}_{n, x x}=\nu^{n-1} F_{n, x x}(E / \nu)
$$

are homogeneous polynomials in $E$ and $\nu$ of degree $n-1$.

Now, consider equation (2.12)

$$
0=\frac{F_{n, x x x}}{2}-2(u-E) F_{n, x}-u_{x} F_{n},
$$

after multiplication by $F_{n}$ and integration, this equation reads

$$
c=\frac{F_{n} F_{n, x x}}{2}-(u-E) F_{n}^{2}-\frac{F_{n, x}^{2}}{4},
$$

where $c$ is a integration constant. By (6.7) we have the following differential relation for the function $g$ :

$$
\frac{1}{2} g g_{x x}-(u-E) g^{2}-\frac{1}{4} g_{x}^{2}=-\frac{1}{4},
$$

since $g_{x}=\left(\sigma_{+}+\sigma_{-}\right) g$ and $g_{x x}=2\left(u-E+\sigma_{+} \sigma_{-}\right) g$.

Now let define the extensions of $\sigma_{+}$and $\sigma_{-}$on $\bar{\Gamma}_{n} \times \mathbb{C}_{x}$ as

$$
\left(\sigma_{+}\right)_{h}=\frac{i \mu \nu^{n-1}+\nu \widehat{F}_{n, x} / 2}{\widehat{F}_{n}}, \quad\left(\sigma_{-}\right)_{h}=\frac{-i \mu \nu^{n-1}+\nu \widehat{F}_{n, x} / 2}{\widehat{F}_{n}},
$$


where we have used previous notation. Notice that the functions $\left(\sigma_{+}\right)_{h}$ and $\left(\sigma_{-}\right)_{h}$ are solutions of the Riccati type equation

$$
\left(\left(\sigma_{ \pm}\right)_{h}\right)^{2}+\left(\left(\sigma_{ \pm}\right)_{x}\right)_{h}=u-E / \nu
$$

Moreover we have that the function

$$
g_{h}=\frac{i \widehat{F}_{n}(E, \nu, x)}{2 \mu \nu^{n-1}}=\frac{1}{\left(\sigma_{-}\right)_{h}-\left(\sigma_{+}\right)_{h}}
$$

is a solution of

$$
\frac{1}{2} g_{h}\left(g_{x x}\right)_{h}-(u-E / \nu) g_{h}^{2}-\frac{1}{4}\left(g_{x}^{2}\right)_{h}=-\frac{1}{4} .
$$

\subsubsection{Transformed Green's functions}

Now, we analyze how Darboux-Crum transformations change Green's functions $g$ and $g_{h}$. For that, we will use solutions of the Riccati type equation (6.4) as a esential tool.

Let $u$ be solution of $s-\mathrm{KdV}_{n}$ equation (2.8). Let $\phi_{1}$ and $\phi_{2}$ be solutions of Schrödinger equation (6.2) for this potential and energy level $E$. Next we consider $\phi_{0}$ a solution of Schrödinger equation for $u$ and $E_{0}$, with $E_{0} \neq E$ and choose as corresponding point of the spectral curve $\left(E_{0}, \mu_{0}\right)$. Recall that after applying a Darboux-Crum transformation with $\phi_{0}$ to $u, \phi_{1}$ and $\phi_{2}$, we get

$$
\operatorname{DT}\left(\phi_{0}\right) u=u-2 \sigma_{0, x}, \quad \operatorname{DT}\left(\phi_{0}\right) \phi_{1}=\phi_{1, x}-\sigma_{0} \phi_{1}, \quad \operatorname{DT}\left(\phi_{0}\right) \phi_{2}=\phi_{2, x}-\sigma_{0} \phi_{2},
$$

where $\sigma_{0}=\left(\log \phi_{0}\right)_{x}$ is a solution of the Riccati equation $\sigma^{2}+\sigma_{x}=u-E_{0}$. By Lemma 6.1 , the function $\sigma^{0}$ equals

$$
\sigma^{0}=\sigma\left(E_{0}, \mu_{0}\right)=\frac{i \mu_{0}+F_{n, x}^{0} / 2}{F_{n}^{0}},
$$

where $F_{n}^{0}=F_{n}\left(E_{0}\right)$, is a solution of the same Riccati equation for $E=E_{0}$. Thus, we conclude that we can perform a Darboux transformation using $\sigma^{0}$ instead of $\sigma_{0}$. The transformed functions

$$
\widetilde{\phi}_{1}=\phi_{1, x}-\sigma^{0} \phi_{1} \quad \text { and } \quad \widetilde{\phi}_{2}=\phi_{2, x}-\sigma^{0} \phi_{2}
$$

are solutions of the Schrödinger equation for potential

$$
\widetilde{u}=u-2 \sigma_{x}^{0} .
$$

Now, we take the functions $\sigma_{1}=\left(\log \phi_{1}\right)_{x}$ and $\sigma_{2}=\left(\log \phi_{2}\right)_{x}$, which are solutions of the Riccati equation (6.4) for $E \neq E_{0}$. Then, by equations (6.5), we get the equalities

$$
\begin{aligned}
& \sigma_{+}-\sigma_{-}=\frac{2 i \mu}{F_{n}}=-\frac{W\left(\phi_{1}, \phi_{2}\right)}{\phi_{1} \phi_{2}}=\frac{\phi_{1, x}}{\phi_{1}}-\frac{\phi_{2, x}}{\phi_{2}}=\sigma_{1}-\sigma_{2}, \\
& \sigma_{+}+\sigma_{-}=\frac{F_{n, x}}{F_{n}}=\frac{\phi_{1} \phi_{2, x}+\phi_{1, x} \phi_{2}}{\phi_{1} \phi_{2}}=\frac{\phi_{1, x}}{\phi_{1}}+\frac{\phi_{2, x}}{\phi_{2}}=\sigma_{1}+\sigma_{2}, \\
& \sigma_{+} \cdot \sigma_{-}=\frac{\phi_{1, x} \phi_{2, x}}{\phi_{1} \phi_{2}}=\frac{\phi_{1, x}}{\phi_{1}} \frac{\phi_{2, x}}{\phi_{2}}=\sigma_{1} \sigma_{2} .
\end{aligned}
$$

Next we define the transformed Green's function

$$
\widetilde{g}(E, \mu, x)=\frac{\widetilde{\phi}_{1} \widetilde{\phi}_{2}}{W\left(\widetilde{\phi}_{1}, \widetilde{\phi}_{2}\right)} .
$$


The relations (6.13)-(6.15) link the Green's functions as follows

$$
\widetilde{g}(E, \mu, x)=\frac{\left(\sigma_{1}-\sigma^{0}\right)\left(\sigma_{2}-\sigma^{0}\right)}{\left(E-E_{0}\right)} \cdot \frac{\phi_{1} \phi_{2}}{W\left(\phi_{1}, \phi_{2}\right)}=\frac{\left(\sigma_{+}-\sigma^{0}\right)\left(\sigma_{-}-\sigma^{0}\right)}{\left(E-E_{0}\right)} g(E, \mu, x) .
$$

Hence we obtain a rational presentation of $\widetilde{g}$ as a consequence of the formulas (6.12) and (6.3). We write this formula in (6.16).

Proposition 6.2. The Green's function associated to the transformed Schrödinger operator explicitly reads

$$
\widetilde{g}(E, \mu, x)=\frac{i\left(\mu^{2}\left(F_{n}^{0}\right)^{2}-\mu_{0}^{2} F_{n}^{2}-i \mu_{0} F_{n}\left(F_{n}^{0} F_{n, x}-F_{n, x}^{0} F_{n}\right)+\frac{\left(F_{n}^{0} F_{n, x}-F_{n, x}^{0} F_{n}\right)^{2}}{4}\right)}{2 \mu\left(E-E_{0}\right) F_{n}\left(F_{n}^{0}\right)^{2}} .
$$

Remark 6.3. Observe that for $E_{0}=0$ the formula (6.16) becomes

$$
\widetilde{g}(E, \mu, x)=\frac{i\left(\mu^{2} f_{n}^{2}-\mu_{0}^{2} F_{n}^{2}-i \mu_{0} F_{n}\left(f_{n} F_{n, x}-f_{n, x} F_{n}\right)+\frac{\left(f_{n} F_{n, x}-f_{n, x} F_{n}\right)^{2}}{4}\right)}{2 \mu E F_{n} f_{n}^{2}} .
$$

We will use the following result from [15].

Proposition 6.4 ([15, Lemma G.1]). Let $u$ be solution of $s-K d V_{n}$ equation, let $\left(E_{0}, \mu_{0}\right)$ and $(E, \mu)$ be two different points of $\Gamma_{n}$. Then the transformed Green's function explicitly reads

$$
\widetilde{g}(E, \mu, x)=\frac{\left(\sigma_{+}-\sigma^{0}\right)\left(\sigma_{-}-\sigma^{0}\right)}{\left(E-E_{0}\right)} \frac{i F_{n}}{2 \mu}=\frac{i \widetilde{F}_{\widetilde{n}}(E, x)}{2 \widetilde{\mu}},
$$

where $\widetilde{F}_{\widetilde{n}}$ is a polynomial in $E$ of degree $\widetilde{n}$ and $\widetilde{\mu}$ is such that $\Gamma_{\widetilde{n}}: \widetilde{\mu}^{2}-\widetilde{R}_{2 \widetilde{n}+1}=0$ for some polynomial $\widetilde{R}_{2 \widetilde{n}+1}(E)$ of degree $2 \widetilde{n}+1$, with $0 \leq \widetilde{n} \leq n+1$.

Next, for the homogeneized Green's function, choose the point of the spectral curve $\left[E_{0}: \mu_{0}:\right.$ $\left.\nu_{0}\right]$. We define the extension of $\sigma^{0}$ on $\bar{\Gamma}_{n} \times \mathbb{C}_{x}$ as

$$
\left(\sigma^{0}\right)_{h}\left(E_{0}, \mu_{0}, \nu_{0}\right)=\frac{i \mu_{0} \nu_{0}^{n-1}+\nu_{0} \widehat{F}_{n, x}^{0} / 2}{\widehat{F}_{n}^{0}},
$$

where $\widehat{F}_{n}^{0}=\widehat{F}_{n}\left(E_{0}, \nu_{0}, x\right)$ for $\widehat{F}_{n}(E, \nu, x)$ defined by $(6.8)$ and $\widehat{F}_{n, x}^{0}=\widehat{F}_{n, x}\left(E_{0}, \nu_{0}, x\right)$, for $\widehat{F}_{n, x}$ defined in (6.10). Notice that when $\nu_{0}=0$ function $\left(\sigma^{0}\right)_{h}$ vanishes. So, whenever $\nu_{0}=0$ we define

$$
\left(\sigma^{0}\right)_{h}\left(E_{0}, \mu_{0}, 0\right):=0, \quad \text { for } \quad\left[E_{0}: \mu_{0}: 0\right] \in \bar{\Gamma}_{n} .
$$

Using above notation we have the following results.

Proposition 6.5. Let assume $C_{0}=R_{2 n+1}(0) \neq 0$. For $E_{0}=0$ and $\mu_{0} \neq 0$, the homogeneized Green's function associated to the transformed Green's function $\widetilde{g}$ for $-\partial_{x x}+\widetilde{u}-E$ explicitly reads

$$
\begin{aligned}
(\widetilde{g})_{h}(E, \mu, \nu, x)= & \frac{i\left(\frac{\nu^{2} \widehat{F}_{n, x x}}{2}+(E-\nu u) \widehat{F}_{n}+\frac{\nu f_{n, x}^{2} \widehat{F}_{n}}{4 f_{n}^{2}}-\frac{\nu^{2} f_{n, x} \widehat{F}_{n, x}}{2 f_{n}}-\frac{\nu C_{0} \widehat{F}_{n}}{f_{n}^{2}}\right)}{2 \mu E \nu^{n-1}} \\
& +\frac{C_{0} \nu_{0}\left(\nu f_{n} \widehat{F}_{n, x}-f_{n, x} \widehat{F}_{n}\right)}{2 \mu E \nu^{n-2} \mu_{0} f_{n}^{2}},
\end{aligned}
$$

where $\widehat{F}_{n}(E, \nu, x)$ is defined by (6.8) and $\widehat{F}_{n, x}(E, \nu, x), \widehat{F}_{n, x x}(E, \nu, x)$ are defined by (6.10). 
Remark 6.6. Formula

$$
\frac{\nu^{2} \widehat{F}_{n, x x}}{2}+(E-\nu u) \widehat{F}_{n}+\frac{\nu f_{n, x}^{2} \widehat{F}_{n}}{4 f_{n}^{2}}-\frac{\nu^{2} f_{n, x} \widehat{F}_{n, x}}{2 f_{n}}-\frac{\nu C_{0} \widehat{F}_{n}}{f_{n}^{2}}
$$

is an homogeneous polynomial in $E$ and $\nu$ of degree $n+1$.

Proof. First, consider the transformed Green's function $\widetilde{g}$ given by (6.16). Then, the homogenized Green's function is obtained by the homogenization process as

$$
\begin{aligned}
(\widetilde{g})_{h}(E, \mu, \nu, x)= & \left(\frac{\left(\sigma_{+}-\sigma^{0}\right)\left(\sigma_{-}-\sigma^{0}\right)}{\left(E-E_{0}\right)} \frac{i F_{n}}{2 \mu}\right)_{h} \\
= & \frac{i\left(\mu^{2} \nu^{2 n-2}\left(\widehat{F}_{n}^{0}\right)^{2}+\frac{\left(\nu \widehat{F}_{n}^{0} \widehat{F}_{n, x}-\nu_{0} \widehat{F}_{n, x}^{0} \widehat{F}_{n}\right)^{2}}{4}\right)}{2 \mu \nu^{n-1}\left(E / \nu-E_{0} / \nu_{0}\right) \widehat{F}_{n}\left(\widehat{F}_{n}^{0}\right)^{2}} \\
& -\frac{i \mu_{0} \nu_{0}^{n-1}\left(\mu_{0} \nu_{0}^{n-1} \widehat{F}_{n}^{2}+i \widehat{F}_{n}\left(\nu \widehat{F}_{n}^{0} \widehat{F}_{n, x}-\nu_{0} \widehat{F}_{n, x}^{0} \widehat{F}_{n}\right)\right)}{2 \mu \nu^{n-1}\left(E / \nu-E_{0} / \nu_{0}\right) \widehat{F}_{n}\left(\widehat{F}_{n}^{0}\right)^{2}},
\end{aligned}
$$

where $\widehat{F}_{n}(E, \nu, x)$ is defined by $(6.8), \widehat{F}_{n, x}(E, \nu, x)$ is defined in $(6.10), \widehat{F}_{n}^{0}=\widehat{F}_{n}\left(E_{0}, \nu_{0}, x\right)$ and $\widehat{F}_{n, x}^{0}=\widehat{F}_{n, x}\left(E_{0}, \nu_{0}, x\right)$. In particular, for $E_{0}=0$, we get

$$
\begin{aligned}
(\widetilde{g})_{h}(E, \mu, \nu, x)= & \frac{i\left(\mu^{2} \nu^{2 n-2} f_{n}^{2}+\frac{\left(\nu f_{n} \widehat{F}_{n, x}-f_{n, x} \widehat{F}_{n}\right)^{2}}{4}\right)}{2 \mu E \nu^{n-2} \widehat{F}_{n} f_{n}^{2}} \\
& -\frac{i \mu_{0}^{2} \widehat{F}_{n}}{2 \mu E \nu^{n-2} \nu_{0}^{2} f_{n}^{2}}+\frac{\mu_{0}\left(\nu f_{n} \widehat{F}_{n, x}-f_{n, x} \widehat{F}_{n}\right)}{2 \mu E \nu^{n-2} \nu_{0} f_{n}^{2}},
\end{aligned}
$$

since $\widehat{F}_{n}\left(0, \nu_{0}, x\right)=\nu_{0}^{n} f_{n}$ and $\widehat{F}_{n, x}\left(0, \nu_{0}, x\right)=\nu_{0}^{n-1} f_{n, x}$. Considering (6.9) we get the following expression

$$
\begin{aligned}
(\widetilde{g})_{h}(E, \mu, \nu, x)= & \frac{i\left(\frac{\nu^{2} \widehat{F}_{n, x x}}{2}+(E-\nu u) \widehat{F}_{n}+\frac{\nu f_{n, x}^{2} \widehat{F}_{n}}{4 f_{n}^{2}}-\frac{\nu^{2} f_{n, x} \widehat{F}_{n, x}}{2 f_{n}}\right)}{2 \mu E \nu^{n-1}} \\
& -\frac{i \mu_{0}^{2} \widehat{F}_{n}}{2 \mu E \nu^{n-2} \nu_{0}^{2} f_{n}^{2}}+\frac{\mu_{0}\left(\nu f_{n} \widehat{F}_{n, x}-f_{n, x} \widehat{F}_{n}\right)}{2 \mu E \nu^{n-2} \nu_{0} f_{n}^{2}} .
\end{aligned}
$$

Moreover, by (6.1) we have that $\mu_{0}^{2}=C_{0} \nu_{0}^{2}$, and then

$$
\begin{aligned}
(\widetilde{g})_{h}(E, \mu, \nu, x)= & \frac{i\left(\frac{\nu^{2} \widehat{F}_{n, x x}}{2}+(E-\nu u) \widehat{F}_{n}+\frac{\nu f_{n, x}^{2} \widehat{F}_{n}}{4 f_{n}^{2}}-\frac{\nu^{2} f_{n, x} \widehat{F}_{n, x}}{2 f_{n}}\right)}{2 \mu E \nu^{n-1}} \\
& -\frac{i C_{0} \widehat{F}_{n}}{2 \mu E \nu^{n-2} f_{n}^{2}}+\frac{C_{0} \nu_{0}\left(\nu f_{n} \widehat{F}_{n, x}-f_{n, x} \widehat{F}_{n}\right)}{2 \mu E \nu^{n-2} \mu_{0} f_{n}^{2}} .
\end{aligned}
$$

And then the result follows.

Proposition 6.7. Let assume $C_{0}=R_{2 n+1}(0)=0$. For $E_{0}=0$ and $\mu_{0} \neq 0$, the homogeneized Green's function associated to the transformed Green's function $\widetilde{g}$ for $-\partial_{x x}+\widetilde{u}-E$ explicitly reads

$$
(\widetilde{g})_{h}(E, \mu, \nu, x)=\frac{i\left(\frac{\nu^{2} \widehat{F}_{n, x x}}{2}+(E-\nu u) \widehat{F}_{n}\right)}{2 \mu E \nu^{n-1}},
$$

where $\widehat{F}_{n}(E, \nu, x)$ is defined by (6.8) and $\widehat{F}_{n, x x}(E, \nu, x)$ is defined in (6.10). 
Remark 6.8. Formula

$$
\frac{\nu^{2} \widehat{F}_{n, x x}}{2}+(E-\nu u) \widehat{F}_{n}
$$

is an homogeneous polynomial in $E$ and $\nu$ of degree $n+1$.

Proof. When $C_{0}=0$ we have that $\nu_{0}=0$ by (6.1), since $\mu_{0} \neq 0$. So, $\left(\sigma^{0}\right)_{h}=0$. Hence, the homogeneized Green's function in this case is

$$
\begin{aligned}
(\widetilde{g})_{h}(E, \mu, \nu, x) & =\frac{\left(\sigma_{+}\right)_{h}\left(\sigma_{-}\right)_{h}}{E / \nu} \frac{i \widehat{F}_{n}}{2 \mu \nu^{n-1}} \\
& =\frac{i\left(\frac{\mu^{2} \nu^{2 n-2}+\nu^{2} \widehat{F}_{n, x}^{2} / 4}{\widehat{F}_{n}}\right)}{2 \mu E \nu^{n-2}}=\frac{i\left(\frac{\nu^{2} \widehat{F}_{n, x x}}{2}+(E-\nu u) \widehat{F}_{n}\right)}{2 \mu E \nu^{n-1}},
\end{aligned}
$$

by $(6.11)$ and (6.9).

\subsection{Darboux-Crum transformations for the spectral curve}

In this subsection we present how Darboux-Crum transformations affect the spectral curve $\Gamma_{n}$. We observe that the action of the transformation DT $\left(\phi_{0}\right)$ strongly depends on the type of point $P$ in the spectral curve we use to construct $\phi_{0}$. In fact, if $P$ is a regular point, the curve associated with the transformed potential is the same; in the other cases the new curve is a blowing-down or a blowing-up of $\Gamma_{n}$.

Theorem 6.9 (I). Let $\left(E_{0}, \mu_{0}\right) \in \Gamma_{n}$ and $u$ be a solution of $s-K d V_{n}$ equation. Let $\phi_{0}$ be a solution of Schrödinger equation for energy $E_{0}$ and potential $u$, i.e., $\phi_{0, x x}=\left(u-E_{0}\right) \phi_{0}$. Let $\widetilde{u}=u-2\left(\log \phi_{0}\right)_{x x}$ be the Darboux-Crum transformation of $u$. Then, $\widetilde{u}$ is a solution of $s-K d V_{\widetilde{n}}$ equation for

$$
\widetilde{n}= \begin{cases}n & \text { if }\left(E_{0}, \mu_{0}\right) \text { is a regular point of } \Gamma_{n}, \\ n-1 & \text { if }\left(E_{0}, \mu_{0}\right) \text { is an affine singular point of } \Gamma_{n} .\end{cases}
$$

Furthermore, the spectral curve associated to $\widetilde{u}$ is $\Gamma_{\widetilde{n}}: \widetilde{\mu}^{2}-\widetilde{R}_{2 \widetilde{n}+1}=0$, with

$$
\widetilde{R}_{2 \widetilde{n}+1}= \begin{cases}R_{2 n+1} & \text { if }\left(E_{0}, \mu_{0}\right) \text { is a regular point of } \Gamma_{n}, \\ \left(E-E_{0}\right)^{-2} R_{2 n+1} & \text { if }\left(E_{0}, \mu_{0}\right) \text { is an affine singular point of } \Gamma_{n} .\end{cases}
$$

The idea of the proof is to compute the Green's function (6.16) associated to $\widetilde{u}$ and interpret the result by means of Lemma 6.4.

Proof. First, we suppose that $\left(E_{0}, \mu_{0}\right)$ is a regular point and $\mu_{0} \neq 0$. In this case, we compute

$$
\left(\sigma_{+}-\sigma^{0}\right)\left(\sigma_{-}-\sigma^{0}\right)=\frac{\mu^{2}\left(F_{n}^{0}\right)^{2}-\mu_{0}^{2} F_{n}^{2}-i \mu_{0} F_{n}\left(F_{n}^{0} F_{n, x}-F_{n, x}^{0} F_{n}\right)+\frac{\left(F_{n}^{0} F_{n, x}-F_{n, x}^{0} F_{n}\right)^{2}}{4}}{F_{n}^{2}\left(F_{n}^{0}\right)^{2}} .
$$

We use Corollaries A.1 and A.2 to rewrite the expressions $F_{n}^{0} F_{n, x}-F_{n, x}^{0} F_{n}$ and $\mu^{2}\left(F_{n}^{0}\right)^{2}-\mu_{0}^{2} F_{n}^{2}$. This yields to the equality

$$
\left(\sigma_{+}-\sigma^{0}\right)\left(\sigma_{-}-\sigma^{0}\right)=\left(E-E_{0}\right) \frac{\frac{P_{n, x}}{2}+F_{n} F_{n}^{0}-P_{n} \sigma^{0}}{F_{n} F_{n}^{0}} .
$$


Finally, we replace this expression in the Green's function (6.16):

$$
\widetilde{g}(E, \mu, x)=\frac{i F_{n}\left(\sigma_{+}-\sigma^{0}\right)\left(\sigma_{-}-\sigma^{0}\right)}{2 \mu\left(E-E_{0}\right)}=\frac{i\left(F_{n}+\frac{P_{n, x}}{2 F_{n}^{0}}-\frac{P_{n} \sigma^{0}}{F_{n}^{0}}\right)}{2 \mu}=\frac{i \widetilde{F}_{\widetilde{n}}}{2 \mu} .
$$

Since $\widetilde{F}_{\widetilde{n}}=F_{n}+\frac{P_{n, x}}{2 F_{n}^{0}}-\frac{P_{n} \sigma^{0}}{F_{n}^{0}}$ is a polynomial in $E$ of degree $n$, by means of Lemma 6.4 , we conclude that $\widetilde{n}=n$ and $\widetilde{\mu}=\mu$. Thus, $\widetilde{R}_{2 \widetilde{n}+1}=R_{2 n+1}$.

Now, we suppose that $\left(E_{0}, \mu_{0}\right)$ is a regular point and $\mu_{0}=0$. In this case, we have that $R_{2 n+1}^{0}=R_{2 n+1}\left(E_{0}\right)=0$ and $R_{2 n+1, E}^{0}=\partial_{E}\left(R_{2 n+1}\right)\left(E_{0}\right) \neq 0$, thus,

$$
\mu^{2}=R_{2 n+1}(E)=\left(E-E_{0}\right) M_{2 n},
$$

where $M_{2 n}(E)$ is a polynomial in $E$ of degree $2 n$ such that $M_{2 n}\left(E_{0}\right) \neq 0$. Hence for $\mu_{0}=0$, $\mu^{2}=\left(E-E_{0}\right) M_{2 n}$ and Corollary A.1, the equality (6.16) becomes

$$
\widetilde{g}(E, \mu, x)=\frac{i\left(\left(E-E_{0}\right) M_{2 n}\left(F_{n}^{0}\right)^{2}+\frac{\left(E-E_{0}\right)^{2} P_{n}^{2}}{4}\right)}{2 \mu\left(E-E_{0}\right) F_{n}\left(F_{n}^{0}\right)^{2}}=\frac{i\left(\frac{M_{2 n}}{F_{n}}+\frac{\left(E-E_{0}\right) P_{n}^{2}}{4 F_{n}\left(F_{n}^{0}\right)^{2}}\right)}{2 \mu} .
$$

Now Corollary A.3 guarantees that

$$
\frac{M_{2 n}}{F_{n}}+\frac{\left(E-E_{0}\right) P_{n}^{2}}{4 F_{n}\left(F_{n}^{0}\right)^{2}}
$$

is a polynomial in $E$ of degree $n$. By Lemma 6.4 , we obtain that $\widetilde{n}=n, \widetilde{\mu}=\mu$ and $\widetilde{R}_{2 \widetilde{n}+1}=R_{2 n+1}$. Therefore, for regular points $\widetilde{R}_{2 \widetilde{n}+1}$ is a polynomial of degree $2 n+1$ in $E$. By Corollary 2.8, we conclude that $\widetilde{u}$ is solution of a s-KdV $\mathrm{K}_{n}$ equation. Thus, a Darboux-Crum transformation with a regular point preserves the spectral curve and the level of the s-KdV hierarchy.

Next, we suppose that $\left(E_{0}, \mu_{0}\right)$ is a singular point of $\Gamma_{n}$, i.e., $\mu_{0}=0, R_{2 n+1}^{0}=R_{2 n+1}\left(E_{0}\right)$ $=0$ and $R_{2 n+1, E}^{0}=\partial_{E}\left(R_{2 n+1}\right)\left(E_{0}\right)=0$, thus,

$$
\mu^{2}=R_{2 n+1}(E)=\left(E-E_{0}\right)^{2} Z_{2 n-1},
$$

where $Z_{2 n-1}(E)$ is a polynomial in $E$ of degree $2 n-1$. Hence for $\mu_{0}=0, \mu^{2}=\left(E-E_{0}\right)^{2} Z_{2 n-1}$ and A.1, the equality (6.16) becomes

$$
\widetilde{g}(E, \mu, x)=\frac{i\left(\left(E-E_{0}\right)^{2} Z_{2 n-1}\left(F_{n}^{0}\right)^{2}+\frac{\left(E-E_{0}\right)^{2} P_{n}^{2}}{4}\right)}{2 \mu\left(E-E_{0}\right) F_{n}\left(F_{n}^{0}\right)^{2}}=\frac{i\left(\frac{Z_{2 n-1}}{F_{n}}+\frac{P_{n}^{2}}{4 F_{n}\left(F_{n}^{0}\right)^{2}}\right)}{2\left(E-E_{0}\right)^{-1} \mu} .
$$

Now Corollary A.4 guarantees that

$$
\frac{Z_{2 n-1}}{F_{n}}+\frac{P_{n}^{2}}{4 F_{n}\left(F_{n}^{0}\right)^{2}}
$$

is a polynomial in $E$ of degree $n$. By Lemma 6.4 , we obtain that $\widetilde{n}=n-1$ and $\widetilde{\mu}=\left(E-E_{0}\right)^{-1} \mu$. Therefore, $\widetilde{R}_{2 \widetilde{n}+1}=\left(E-E_{0}\right)^{-2} R_{2 n+1}$ is a polynomial of degree $2 n-1$ in $E$. By Corollary 2.8 , we conclude that $\widetilde{u}$ is solution of a s-KdV $V_{n-1}$ equation. So, a Darboux-Crum transformation with a singular point induces a blow-up in the spectral curve in this singular point and reduces the level of the s-KdV hierarchy in one. 
Next, we will proceed to establish the situation at the point of infinity $P_{\infty}=[0: 1: 0]$ of the spectral curve. For that, we will need to work with the Zariski closure in $\mathbb{P}^{2}$ of the spectral curve to understand its behaviour under Darboux transformations for the energy level $E_{0}=0$. In addition, we will use the blowing-up map in $\mathbb{P}^{2}$ to control the $\mathrm{KdV}$ level of the transformed potential $\widetilde{u}$.

Let $\pi: \widetilde{\mathbb{P}^{2}} \rightarrow \mathbb{P}^{2}$ be the blowing-up of $\mathbb{P}^{2}$ with center $[0: 0: 1]$. Hence, if $[E: \mu: \nu]$ are homegeneous coordinates in $\mathbb{P}^{2}$, then the new ones are denoted by $[\widetilde{E}: \widetilde{\mu}: \widetilde{\nu}$, and $\pi$ is given by

$$
E=\widetilde{E}, \quad \mu E=\widetilde{\mu}, \quad \nu=\widetilde{\nu} .
$$

Theorem 6.10 (II). Let $P_{\infty}=[0: 1: 0]$ be the infinity point of $\bar{\Gamma}_{n}$, and $u$ a solution of the $s$ $K d V_{n}$ equation. Let $\phi_{0}$ be a solution of Schrödinger equation for $P_{\infty}\left(\right.$ in particular $\left.E_{0}=0\right)$ and potential $u$, i.e., $\phi_{0, x x}-u \phi_{0}=0$. Let $\widetilde{u}=u-2\left(\log \phi_{0}\right)_{x x}$ be the Darboux-Crum transformation of $u$. Then, $\widetilde{u}$ is solution of the $s-K d V_{n+1}$ equation. Futhermore, the spectral curve associated to $\widetilde{u}$ is $\Gamma_{n+1}: \widetilde{\mu}^{2}-\widetilde{R}_{2 n+3}(E)=0$, with $\widetilde{R}_{2 n+3}=E^{2} R_{2 n+1}(E)$.

Proof. First, consider the homogeneized Green's function associated to the transformed Green's function $\widetilde{g}$. Then, by Propositions 6.5 and $6.7,(\widetilde{g})_{h}$ is a well defined rational function on $\bar{\Gamma}_{n}$. But also we have

$$
(\widetilde{g})_{h}=G_{h} \circ \pi \text { on the spectral curve. }
$$

Moreover $G_{h}$ is a Green function for the curve defined by $\widetilde{\mu}^{2}-\widetilde{R}_{2 n+3}(\widetilde{E})=0$, where $\widetilde{R}_{2 n+3}(\widetilde{E})=$ $E^{2} R_{2 n+1}(E)$; that is, for $\Gamma_{n+1}$, the strict transform of $\Gamma_{n}$. Observe that $\widetilde{R}_{2 n+3}=E^{2} R_{2 n+1}$ is a polynomial of degree $2 n+3$ in $E$. Then, by Corollary 2.8, we conclude that $\widetilde{u}$ is solution of a $\mathrm{s}-\mathrm{KdV}_{n+1}$ equation.

Finally we can rewrite Theorems 6.9 and 6.10 to establish how the spectral curve $\bar{\Gamma}_{n}$ behaves under Darboux-Crum transformations.

Theorem 6.11. Let $P=\left[E_{0}: \mu_{0}: \nu_{0}\right]$ be a point in $\bar{\Gamma}_{n}$, and $u$ a solution of $s-K d V_{n}$ equation. Let $\phi_{0}$ be a solution of Schrödinger equation for $E_{0}$ and potential $u$, say $\phi_{0, x x}=\left(u-E_{0}\right) \phi_{0}$. Consider $\widetilde{u}=u-2\left(\log \phi_{0}\right)_{x x}$ the Darboux-Crum transformation of $u$. Then, $\widetilde{u}$ is solution of $s-K d V_{\widetilde{n}}$ equation for

$$
\widetilde{n}= \begin{cases}n+1 & \text { if } P=[0: 1: 0] \\ n & \text { if } P \text { is a regular point of } \Gamma_{n}, \\ n-1 & \text { if } P \text { is an affine singular point of } \Gamma_{n} .\end{cases}
$$

Futhermore, the spectral curve associated to $\widetilde{u}$ is $\Gamma_{\widetilde{n}}: \widetilde{\mu}^{2}-\widetilde{R}_{2 \widetilde{n}+1}=0$, with

$$
\widetilde{R}_{2 \widetilde{n}+1}= \begin{cases}E^{2} R_{2 n+1} & \text { if } P=[0: 1: 0] \\ R_{2 n+1} & \text { if } P \text { is a regular point of } \Gamma_{n}, \\ \left(E-E_{0}\right)^{-2} R_{2 n+1} & \text { if } P \text { is an affine singular point of } \Gamma_{n} .\end{cases}
$$

Example 6.12. Next we apply the previous theorem to a rational s-KdV $\mathrm{V}_{2}$ potential.

Take the $s-K_{2}$ potential $u=\frac{6}{x^{2}}$ in the Schrödinger equation (6.2). The spectral curve associated to this potential is $\Gamma_{2}: \mu^{2}-E^{5}=0$. When $E=0$, we have the fundamental solutions $\phi_{1}=x^{-2}$ and $\phi_{2}=x^{3}$. We consider the Darboux transformations of $u$ with these solutions

$$
\operatorname{DT}\left(\phi_{1}\right) u=u-2\left(\log \phi_{1}\right)_{x x}=\frac{2}{x^{2}}=\widetilde{u}_{1} \quad \text { and } \quad \operatorname{DT}\left(\phi_{2}\right) u=u-2\left(\log \phi_{2}\right)_{x x}=\frac{12}{x^{2}}=\widetilde{u}_{3} .
$$


We have that potential $\widetilde{u}_{1}$ is a solution of $\mathrm{s}-\mathrm{KdV}_{1}$ equation. It is well known that the spectral curve associated to this potential is $\Gamma_{1}: \mu^{2}-E^{3}=0$, the blowing-up of $\Gamma_{2}$ at $(0,0)$. Furthermore, potential $\widetilde{u}_{3}$ is a solution of s- $\mathrm{KdV}_{3}$ equation, and its associated spectral curve $\Gamma_{3}$ is the blowingdown of $\Gamma_{2}$, that is $\Gamma_{3}: \mu^{2}-E^{7}=0$.

Now, we take a regular value of $E$ in $\Gamma_{2}$, for instance, $E=-1$. Then, a solution of the Schrödinger equation (6.2) for this value of $E$ is $\phi^{+}=\frac{e^{x}\left(x^{2}-3 x+3\right)}{x^{2}}$. The Darboux transformation of $u$ with this solution reads

$$
\mathrm{DT}\left(\phi^{+}\right) u=u-2\left(\log \phi^{+}\right)_{x x}=\frac{6(x-1)\left(x^{3}-3 x^{2}+3 x-3\right)}{x^{2}\left(x^{2}-3 x+3\right)^{2}}=\widetilde{u} .
$$

Then this transformed potential is a solution of $\mathrm{s}-\mathrm{KdV}_{2}$ equation and the spectral curve associated to this potential is still $\Gamma_{2}: \mu^{2}-E^{5}=0$.

We sum up this example in the following diagram:

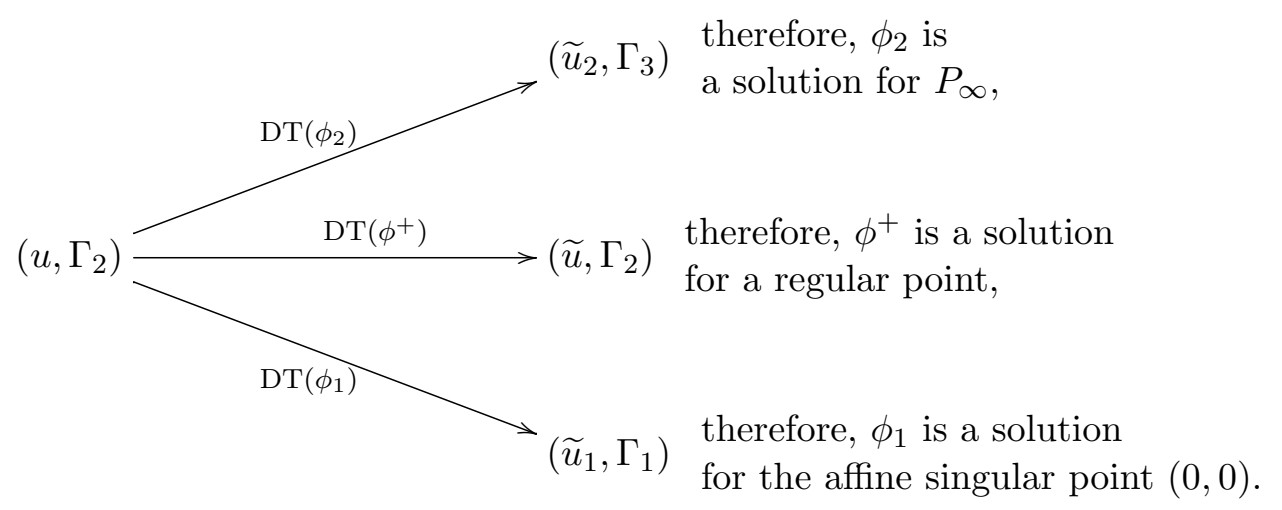

Remark 6.13. The importance of Theorem 6.11 lies in the fact that we need to introduce the homogenized Green's function to state it. This new function is the essential tool that allows us to include in our study the point of infinity $P_{\infty}$ of the affine curve $\Gamma_{n}$. As far as we know, this is a new approach to the understanding of the spectral curve under Darboux transformations.

Similar problems to our result 6.11 were treated by several authors, see [13, Theorem 5] and [15, Theorem G.2]. In [13], F. Ehlers and H. Knörrer studied the action of the Darboux transformations on the spectral curves by means of the eigenfunctions of the centralizer of the Schrödinger operator.

\subsection{Spectral curves and KdV hierarchy in $1+1$ dimensions}

In this section we will show how the points of the spectral curves in the stationary setting are related with the solutions of the Schrödinger operator with rational potential in the $1+1 \mathrm{KdV}$ hierarchy.

Recall that the rational soliton $u_{r, n}$ restricted to $t_{r}=0$ is the well known $n$-soliton $u_{n}^{(0)}(x)=$ $n(n+1) x^{-2}$. Let $\Gamma_{n}$ be its affine spectral curve. This complex plane curve has a defining equation

$$
p_{n}(E, \mu)=\mu^{2}-E^{2 n+1} .
$$

Our goal was to obtain the algebraic structure of a fundamental matrix of the Schrödinger operator $-\partial_{x}^{2}+u_{r, n}-E$ by means of the system (4.1). For this purpose we need to use a parametric representation of the spectral curve $\Gamma_{n}$. Observe that $\Gamma_{n}$ is a rational singular plane curve, nevertheless we can have a global parametrization in the sense given in [3]. In fact, we have considered the parametrization

$$
\chi(\lambda)=\left(-\lambda^{2}, i \lambda^{2 n+1}\right)
$$


and then $E=-\lambda^{2}$ as was taken since Section 4. Observe that the unique affine singular point of the spectral curve is reached for $\lambda=0$. Hence, whenever $\lambda \neq 0$ we obtain regular points on $\Gamma_{n}$ and we can get the desired description of the fundamental matrix $\mathcal{B}_{n, \lambda}^{(r)}$ as is given in Theorem 4.6. On the other hand, at the singular point $\chi(0)=(0,0)$ the fundamental matrix for the system (4.1) must be obtained in a specific way, see Theorem 4.1.

The fundamental solutions $\phi_{1, r, n}\left(x, t_{r}\right), \phi_{2, r, n}\left(x, t_{r}\right)$ obtained in Theorem 4.1 were used as source to perform Darboux transformations. In particular, for $t_{r}=0$, we get the functions

$$
\phi_{1, n}^{(0)}(x)=\phi_{1, r, n}\left(x, t_{r}=0\right), \quad \phi_{2, n}^{(0)}(x)=\phi_{2, r, n}\left(x, t_{r}=0\right)
$$

and the corresponding potentials are transformed as is indicated in the following diagram:

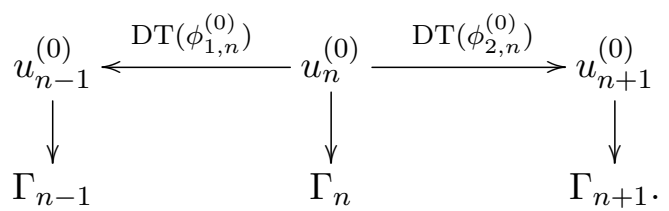

This situation is a particular case of a more general one that has been obtained in Theorem 6.11. The diagram (6.17) has its time dependent counterpart (see (4.3) and (5.3))

$$
u_{r, n-1} \stackrel{\mathrm{DT}\left(\phi_{1, r, n}\right)}{\longleftarrow} u_{r, n} \stackrel{\mathrm{DT}\left(\phi_{2, r, n}\right)}{\longrightarrow} u_{r, n+1} .
$$

The fundamental matrix $\mathcal{B}_{n, 0}^{(r)}$ associated to the functions $\phi_{1, r, n}$ and $\phi_{2, r, n}$ can not be changed by the same Darboux transformations used for the potentials since there is a loss of independent solutions; in fact we have the following diagram:

$$
\begin{aligned}
& \phi_{1, r, n} \stackrel{\mathrm{DT}\left(\phi_{2, r, n}\right)}{\longrightarrow} \phi_{1, r, n+1}, \\
& \phi_{2, r, n-1} \stackrel{\mathrm{DT}\left(\phi_{1, r, n}\right)}{\longleftarrow} \phi_{2, r, n} .
\end{aligned}
$$

On the other hand, whenever the point on the spectral curve is a regular point, that is $\lambda \neq 0$, we have obtained the behaviour of the fundamental matrices $\mathcal{B}_{j, \lambda}^{(r)}$, for $j=n-1, n, n+1$, as it is encoded in the following diagram:

$$
\begin{aligned}
& \phi_{r, n-1}^{+} \stackrel{\mathrm{DT}\left(\phi_{1, r, n}\right)}{\longleftarrow} \phi_{r, n}^{+} \stackrel{\mathrm{DT}\left(\phi_{2, r, n}\right)}{\longrightarrow} \phi_{r, n+1}^{+}, \\
& \phi_{r, n-1}^{-} \stackrel{\mathrm{DT}\left(\phi_{1, r, n}\right)}{\longleftarrow} \phi_{r, n}^{-} \stackrel{\mathrm{DT}\left(\phi_{2, r, n}\right)}{\longrightarrow} \phi_{r, n+1}^{-} .
\end{aligned}
$$

All these situations are reflected in the time dependent frame coming from the stationary one, as we have seen. In particular, in the lack of specialization process from $\mathcal{B}_{n, \lambda}^{(r)}$ to $\mathcal{B}_{n, 0}^{(r)}$. According to Theorem 4.8, we have that $\operatorname{det} \mathcal{B}_{n, \lambda}^{(r)}=-2 \lambda^{2 n+1}$, whereas we have $\operatorname{det} \mathcal{B}_{n, 0}^{(r)}=2 n+1$.

Remark 6.14. We notice then that, despite functions $\phi_{1, n}^{(0)}$ and $\phi_{2, n}^{(0)}$ are fundamental solutions of the Schrödinger equation for $E=0$, they are not solutions for the same point of the spectral curve. Therefore, for each singular point of this spectral curve we can only compute one fundamental solution by means of Darboux transformations.

On the other hand, the stationary functions corresponding to $\phi_{r, n}^{+}$and $\phi_{r, n}^{-}$, namely,

$$
\left(\phi_{n}^{+}\right)^{(0)}(x, \lambda)=\phi_{r, n}^{+}\left(x, \lambda, t_{r}=0\right) \quad \text { and } \quad\left(\phi_{n}^{-}\right)^{(0)}(x, \lambda)=\phi_{r, n}^{-}\left(x, \lambda, t_{r}=0\right),
$$


are fundamental solutions at regular points of the spectral curve, since they are solutions of the Schrödinger equation for $E \neq 0$. In fact, one of them, say $\left(\phi_{n}^{+}\right)^{(0)}(x, \lambda)$, is a solution for the point $(E, \mu)$, and the other one, say $\left(\phi_{n}^{-}\right)^{(0)}(x, \lambda)$, is a solution for the conjugated point $(E,-\mu)$ of the spectral curve. Then, for each value of $E=-\lambda^{2}$, the fundamental matrix $\mathcal{B}_{n, \lambda}^{(r)}$ shows the solutions at conjugated points on the corresponding spectral curve.

Next we have computed an explicit example to illustrate the relationship between spectral curves and KdV hierarchy in $1+1$ dimensions for rational solitons.

Example 6.15. Consider the case $r=1$ and $n=2$. Let $u_{1,2}\left(x, t_{1}\right)=\frac{6 x\left(x^{3}-6 t_{1}\right)}{\left(x^{3}+3 t_{1}\right)^{2}}$ be the $\mathrm{KdV}_{1}$ rational soliton obtained by taking $\left(\tau_{2}, \tau_{3}\right)=\left(3 t_{1}, 0\right)$. Then, the corresponding stationary potential is given by $u_{2}^{(0)}(x)=u_{1,2}^{(0)}(x)=u_{1,2}\left(x, t_{r}=0\right)=\frac{6}{x^{2}}$ (see Lemma 2.9). Its spectral curve is $\Gamma_{2}: p_{2}(E, \mu)=\mu^{2}-E^{5}$.

Futhermore, the stationary Schrödinger operator presents two types of solutions a priori. In fact, when $E=0$, the solutions are

$$
\phi_{1,2}^{(0)}:=\phi_{1,1,2}\left(x, t_{r}=0\right)=x^{-2}, \quad \phi_{2,2}^{(0)}:=\phi_{2,1,2}\left(x, t_{r}=0\right)=x^{3},
$$

where

$$
\phi_{1,1,2}\left(x, t_{r}\right)=\frac{x}{x^{3}+3 t_{1}}, \quad \phi_{2,1,2}\left(x, t_{r}\right)=\frac{x^{6}+15 x^{3} t_{1}-45 t_{1}^{2}}{x^{3}+3 t_{1}}
$$

as they were computed in Example 4.4. In this case, we have the following diagram:

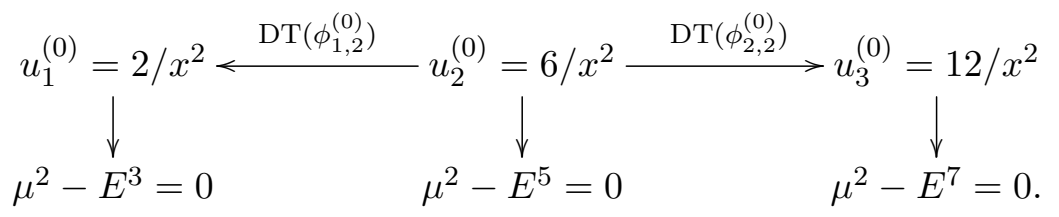

When energy $E \neq 0$, in Example 5.4 we have computed the solutions

$$
\phi_{1,2}^{+}=e^{\lambda x-\lambda^{3} t_{1}} \frac{\lambda^{2} x^{3}-3 \lambda x^{2}+3 x+3 \lambda^{2} t_{1}}{x^{3}+3 t_{1}}, \quad \phi_{1,2}^{-}=e^{-\lambda x+\lambda^{3} t_{1}} \frac{\lambda^{2} x^{3}+3 \lambda x^{2}+3 x+3 \lambda^{2} t_{1}}{x^{3}+3 t_{1}},
$$

where we have adjusted parameters $\tau_{2}^{+}=3 \lambda^{2} t_{1}=\tau_{2}^{-}$. Next, take $t_{1}=0$ to obtain

$$
\begin{aligned}
& \phi_{2}^{+}(x, \lambda)=\phi_{1,2}^{+}\left(x, t_{r}=0, \lambda\right)=e^{\lambda x} \frac{\lambda^{2} x^{3}-3 \lambda x^{2}+3 x}{x^{3}}, \\
& \phi_{2}^{-}(x, \lambda)=\phi_{1,2}^{-}\left(x, t_{r}=0, \lambda\right)=e^{-\lambda x} \frac{\lambda^{2} x^{3}+3 \lambda x^{2}+3 x}{x^{3}} .
\end{aligned}
$$

These functions are solutions of the Schrödinger operator for the stationary potential $u_{2}^{(0)}=6 / x^{2}$ whenever $E \neq 0$. Observe that $\phi_{2}^{+}(x, 0)=3 / x^{2}=\phi_{2}^{-}(x, 0)$, and then they are no longer independent (see Example 4.10 for the general case).

Next, we will show how the Darboux transformations act on time dependent potentials and solutions. First recall that for any potential $u$, we have defined the Darboux transformation as

$$
\operatorname{DT}\left(\phi_{i, r, n}\right) u=u-2\left(\log \phi_{i, r, n}\right)_{x x}, \quad i=1,2 .
$$

Next, we perform the Darboux transformations by means of $\phi_{1,1,2}$ and $\phi_{2,1,2}$ to our initial potential $u_{1,2}$. In these cases we have obtained

$$
u_{1,1}=\frac{2}{x^{2}} \stackrel{\mathrm{DT}\left(\phi_{1,1,2}\right)}{\longleftarrow} u_{1,2}=\frac{6 x\left(x^{3}-6 t_{1}\right)}{\left(x^{3}+3 t_{1}\right)^{2}} \stackrel{\mathrm{DT}\left(\phi_{2,1,2}\right)}{\longrightarrow} u_{1,3}=\frac{6 x\left(2 x^{9}+675 x^{3} t_{1}^{2}+1350 t_{1}^{3}\right)}{\left(x^{6}+15 x^{3} t_{1}-45 t_{1}^{2}\right)^{2}} .
$$


Then, we must consider the Schrödinger operators

$$
-\partial_{x}^{2}+u_{1, j}\left(x, t_{1}\right)-E, \quad j=1,2,3 .
$$

Their solutions $\phi_{1, j}^{+}$and $\phi_{1, j}^{-}$were given in Example 5.4.

It should be noted that if the energy is not zero, these solutions inherit the same behaviour as their corresponding potentials when the Darboux transformations $\operatorname{DT}\left(\phi_{1,1,2}\right)$ and $\operatorname{DT}\left(\phi_{2,1,2}\right)$ act on them. Hence we obtain the following diagram:

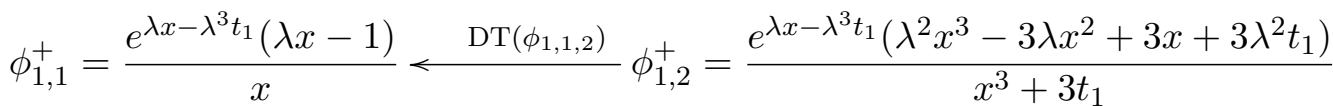

$$
\begin{aligned}
& \stackrel{\mathrm{DT}\left(\phi_{2,1,2}\right)}{\longrightarrow} \phi_{1,3}^{+}=\frac{e^{\lambda x-\lambda^{3} t_{1}} Q_{3}^{+}\left(\lambda, x, t_{1}\right)}{x^{6}+15 x^{3} t_{1}-45 t_{1}^{2}},
\end{aligned}
$$



$$
\begin{aligned}
& \stackrel{\mathrm{DT}\left(\phi_{2,1,2}\right)}{\longrightarrow} \phi_{1,3}^{-}=\frac{e^{-\lambda x+\lambda^{3} t_{1}} Q_{3}^{-}\left(\lambda, x, t_{1}\right)}{x^{6}+15 x^{3} t_{1}-45 t_{1}^{2}},
\end{aligned}
$$

where

$$
\begin{aligned}
Q_{3}^{+}\left(\lambda, x, t_{1}\right)= & \lambda^{3} x^{6}-6 \lambda^{2} x^{5}+15 \lambda x^{4}-15 x^{3}+15 \lambda^{3} x^{3} t_{1}-45 \lambda^{2} x^{2} t_{1}+45 \lambda x t_{1} \\
& -45 \lambda^{3} t_{1}^{2}-45 t_{1}, \\
Q_{3}^{-}\left(\lambda, x, t_{1}\right)= & \lambda^{3} x^{6}+6 \lambda^{2} x^{5}+15 \lambda x^{4}+15 x^{3}+15 \lambda^{3} x^{3} t_{1}+45 \lambda^{2} x^{2} t_{1}+45 \lambda x t_{1} \\
& -45 \lambda^{3} t_{1}^{2}+45 t_{1} .
\end{aligned}
$$

The zero energy case is essentially different from the point of view of the Darboux transformations. We only can partially obtain the previous diagram:

$$
\begin{gathered}
\phi_{1,1,2}=\frac{x}{x^{3}+3 t_{1}} \stackrel{\mathrm{DT}\left(\phi_{2,1,2}\right)}{\longrightarrow} \phi_{1,1,3}=\frac{x^{3}+3 t_{1}}{x^{6}+15 x^{3} t_{1}-45 t_{1}^{2}} \\
\phi_{2,1,1}=\frac{x^{3}+3 t_{1}}{x} \longleftarrow \stackrel{\mathrm{DT}\left(\phi_{1,1,2}\right)}{\longleftarrow} \phi_{2,1,2}=\frac{x^{6}+15 x^{3} t_{1}-45 t_{1}^{2}}{x^{3}+3 t_{1}} .
\end{gathered}
$$

To compute fundamental matrices associated to $u_{1,1}$ and $u_{1,3}$ we have to use Theorem 4.1 (see Example 4.4).

\section{Differential Galois groups}

In this section we study the Picard-Vessiot extensions of the differential systems (4.2) and (4.9), obtained for energy levels $E=0$ and $E \neq 0$ respectively. We recall that the base differential field is $K_{r}=\mathbb{C}\left(x, t_{r}\right)$ with field of constants $\mathbb{C}$.

We point out that the behaviour that they present depend strongly on the affine point $P=(E, \mu)$ of the corresponding spectral curve. They present a similar behaviour when the point $P=(E, \mu)$ is a regular point of $\Gamma_{n}$.

A fundamental matrix for $E=0$ can be also computed. However, it is not obtained by a specialization process from the fundamental matrix obtained for a regular point.

We obtain the Picard-Vessiot extensions given by $\mathcal{B}_{n, 0}^{(r)}$ and $\mathcal{B}_{n, \lambda}^{(r)}$ and compute their corresponding differential Galois group, say $\mathcal{G}_{n, 0}^{(r)}$ and $\mathcal{G}_{n, \lambda}^{(r)}$ respectively. 


\subsection{Case $E=0$}

For this case we have the fundamental matrix

$$
\mathcal{B}_{n, 0}^{(r)}=\left(\begin{array}{cc}
\phi_{1, r, n} & \phi_{2, r, n} \\
\phi_{1, r, n, x} & \phi_{2, r, n, x}
\end{array}\right)
$$

where $\phi_{1, r, n}, \phi_{1, r, n, x}, \phi_{2, r, n}, \phi_{2, r, n, x}$ are rational functions in $x, t$, hence they are in $K_{r}$. So, the Picard-Vessiot field is again $K_{r}$. Thus, the differential Galois group is the trivial group, $\mathcal{G}_{n, 0}^{(r)}=\left\{\mathrm{id}_{2}\right\}$.

\subsection{Case $E \neq 0$}

In this case, we compute the differential extension given for each value of $\lambda \neq 0$. For this, we fix a value of $\lambda$ different from zero, $\lambda=\lambda_{0}$, then the point $P=\left(E_{0}, \mu_{0}\right)$ is a regular point of $\Gamma_{n}$, that is $E_{0} \neq 0$. The fundamental matrix is

$$
\mathcal{B}_{n, \lambda_{0}}^{(r)}=\left(\begin{array}{cc}
\phi_{r, n}^{+}\left(\lambda_{0}\right) & \phi_{r, n}^{-}\left(\lambda_{0}\right) \\
\phi_{r, n, x}^{+}\left(\lambda_{0}\right) & \phi_{r, n, x}^{-}\left(\lambda_{0}\right)
\end{array}\right),
$$

for $\phi_{r, n}^{+}\left(\lambda_{0}\right), \phi_{r, n, x}^{+}\left(\lambda_{0}\right), \phi_{r, n}^{-}\left(\lambda_{0}\right)$ and $\phi_{r, n, x}^{-}\left(\lambda_{0}\right) \in K_{r}\left(\eta_{r}\right)$, with $\eta_{r}=e^{\lambda_{0} x+(-1)^{r} \lambda_{0}^{2 r+1} t_{r}}$. Then, the Picard-Vessiot field is $L_{r}=K_{r}\left(\eta_{r}\right)$.

To compute the differential Galois group $\mathcal{G}_{n, \lambda_{0}}^{(r)}$ in this case, we just have to compute the action of $\mathcal{G}_{n, \lambda_{0}}^{(r)}$ on $\eta_{r}$. For this, let $\sigma$ in $\mathcal{G}_{n, \lambda_{0}}^{(r)}$ be an automorphism of the differential Galois group, then

$$
\begin{aligned}
\left(\frac{\sigma\left(\eta_{r}\right)}{\eta_{r}}\right)_{x} & =\frac{\sigma\left(\lambda_{0} \eta_{r}\right)-\lambda_{0} \sigma\left(\eta_{r}\right)}{\eta_{r}}=\frac{\lambda_{0} \sigma\left(\eta_{r}\right)-\lambda_{0} \sigma\left(\eta_{r}\right)}{\eta_{r}}=0, \\
\left(\frac{\sigma\left(\eta_{r}\right)}{\eta_{r}}\right)_{t_{r}} & =\frac{\sigma\left((-1)^{r} \lambda_{0}^{2 r+1} \eta_{r}\right)-(-1)^{r} \lambda_{0}^{2 r+1} \sigma\left(\eta_{r}\right)}{\eta_{r}} \\
& =\frac{(-1)^{r} \lambda_{0}^{2 r+1} \sigma\left(\eta_{r}\right)-(-1)^{r} \lambda_{0}^{2 r+1} \sigma\left(\eta_{r}\right)}{\eta_{r}}=0 .
\end{aligned}
$$

Therefore $\frac{\sigma\left(\eta_{r}\right)}{\eta_{r}}$ is a constant in $K_{r}$. Hence $\sigma\left(\eta_{r}\right)=c \cdot \eta_{r}$ for some $c \in \mathbb{C}$. As a consequence we get that, for each $\lambda_{0}$ and every $n$, the differential Galois group is isomorphic to the multiplicative group, say

$$
\mathcal{G}_{n, \lambda_{0}}^{(r)} \simeq G_{m}=\left\{\left(\begin{array}{cc}
c & 0 \\
0 & c^{-1}
\end{array}\right): c \in \mathbb{C}^{*}\right\}
$$

Remark 7.1. Since the Galois groups $\mathcal{G}_{n, \lambda_{0}}^{(r)}$ are obtained for a particular value of $\lambda$ by especialization process, they do not depend on $\lambda$. For a spectral study of the Picard-Vessiot extensions see $[21]$.

\subsection{Global behaviour of the differential Galois groups}

Let us consider the family of linear algebraic groups $\left\{\mathcal{G}_{n, \lambda}^{(r)}\right\}_{\lambda \in \mathbb{C}}$. Then for each point in $\Gamma_{n}$ we have found a linear algebraic group. As a result of our constructions we have a sheave structure of groups on the regular points of $\Gamma_{n}$

$$
\Gamma_{n} \backslash \operatorname{Sing}\left(\Gamma_{n}\right) \ni\left(-\lambda^{2}, i \lambda^{2 n+1}\right) \longrightarrow \mathcal{G}_{n, \lambda}^{(r)} .
$$


For each $\lambda \in \mathbb{C}$, the situation is encoded in the following diagram

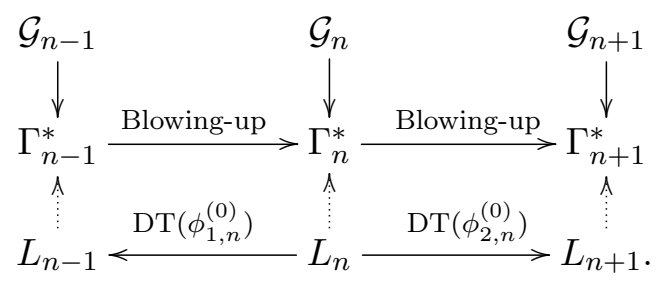

We observe the invariance of the Galois groups with respect to:

- The time of each level $r$ of the KdV hierarchy once it have been adjusted to the level of the $K d V$ hierarchy. Observe that we are constructing the field of coefficients $K_{r}$.

- Generic values of the spectral parameter, i.e., moving along the regular points of the spectral curve.

- Darboux transformations.

\section{A Auxiliary results}

We establish a series of easy corollaries of the result of Proposition 6.2. They are necessary in the Section 6.2. We use the same notation as in Section 6.1.

Corollary A.1. We have

$$
F_{n}^{0} F_{n, x}-F_{n, x}^{0} F_{n}=\left(E-E_{0}\right) P_{n},
$$

where $P_{n}$ is a polynomial in $E$ of degree at most $n-1$. In particular for $E_{0}=0$ we obtain

$$
f_{n} F_{n, x}-f_{n, x} F_{n}=E P_{n} .
$$

Proof. Since $F_{n}=\sum_{l=0}^{n} f_{n-l} E^{l}$ and $F_{n}^{0}=\sum_{l=0}^{n} f_{n-l} E_{0}^{l}$, we have that

$$
\begin{aligned}
F_{n}^{0} F_{n, x}-F_{n, x}^{0} F_{n} & =\sum_{i, j=0}^{n} f_{n-i} f_{n-j, x} E_{0}^{i} E^{j}-\sum_{i, j=0}^{n} f_{n-i} f_{n-j, x} E_{0}^{j} E^{i} \\
& =\sum_{\substack{i, j=0 \\
i \neq j}}^{n}\left(E_{0}^{i} E^{j}-E_{0}^{j} E^{i}\right) f_{n-i} f_{n-j, x} .
\end{aligned}
$$

We factor the term $E_{0}^{i} E^{j}-E_{0}^{j} E^{i}$ :

$$
E_{0}^{i} E^{j}-E_{0}^{j} E^{i}=\left(E-E_{0}\right)\left(E E_{0}\right)^{\min (i, j)}(-1)^{\operatorname{sign}(i, j)}\left(\sum_{k=0}^{|j-i|-1} E^{k} E_{0}^{|j-i|-1-k}\right),
$$

and replace it in (A.1). We get

$$
\begin{aligned}
& F_{n}^{0} F_{n, x}-F_{n, x}^{0} F_{n}= \\
& \quad=\left(E-E_{0}\right) \sum_{\substack{i, j=0 \\
i \neq j}}^{n}\left(E E_{0}\right)^{\min (i, j)}(-1)^{\operatorname{sign}(i, j)}\left(\sum_{k=0}^{|j-i|-1} E^{k} E_{0}^{|j-i|-1-k}\right) f_{n-i} f_{n-j, x} \\
& \quad=\left(E-E_{0}\right) P_{n},
\end{aligned}
$$

for $P_{n}$ a polynomial in $E$ of degree at most $n-1$, as it is stated. 
Corollary A.2. We have

$$
\mu^{2}\left(F_{n}^{0}\right)^{2}-\mu_{0}^{2} F_{n}^{2}=\left(E-E_{0}\right)\left(\frac{F_{n} F_{n}^{0} P_{n, x}}{2}+F_{n}^{2}\left(F_{n}^{0}\right)^{2}-\frac{P_{n}\left(F_{n} F_{n, x}^{0}+F_{n, x} F_{n}^{0}\right)}{4}\right),
$$

where $P_{n}$ is the polynomial obtained in Corollary A.1. In particular for $E_{0}=0$ we obtain

$$
\mu^{2} f_{n}^{2}-\mu_{0}^{2} F_{n}^{2}=E\left(\frac{F_{n} f_{n} P_{n, x}}{2}+F_{n}^{2}\left(f_{n}\right)^{2}-\frac{P_{n}\left(F_{n} f_{n, x}+F_{n, x} f_{n}\right)}{4}\right) .
$$

Proof. By (2.13) we have

$$
\begin{aligned}
& \mu^{2}=R_{2 n+1}=\frac{F_{n} F_{n, x x}}{2}-(u-E) F_{n}^{2}-\frac{F_{n, x}^{2}}{4}, \\
& \mu_{0}^{2}=R_{2 n+1}\left(E_{0}\right)=\frac{F_{n}^{0} F_{n, x x}^{0}}{2}-\left(u-E_{0}\right)\left(F_{n}^{0}\right)^{2}-\frac{\left(F_{n, x}^{0}\right)^{2}}{4} .
\end{aligned}
$$

Hence,

$$
\begin{aligned}
\mu^{2}\left(F_{n}^{0}\right)^{2}-\mu_{0}^{2} F_{n}^{2}= & \frac{F_{n} F_{n}^{0}}{2}\left(F_{n, x x} F_{n}^{0}-F_{n, x x}^{0} F_{n}\right)+\frac{F_{n}^{2}\left(F_{n, x}^{0}\right)^{2}-F_{n, x}^{2}\left(F_{n}^{0}\right)^{2}}{4}+\left(E-E_{0}\right) F_{n}^{2}\left(F_{n}^{0}\right)^{2} \\
= & \frac{F_{n} F_{n}^{0}}{2}\left(F_{n, x x} F_{n}^{0}-F_{n, x x}^{0} F_{n}\right)+\left(E-E_{0}\right) F_{n}^{2}\left(F_{n}^{0}\right)^{2} \\
& +\frac{\left(F_{n} F_{n, x}^{0}-F_{n, x} F_{n}^{0}\right)\left(F_{n} F_{n, x}^{0}+F_{n, x} F_{n}^{0}\right)}{4}
\end{aligned}
$$

As $F_{n}^{0} F_{n, x x}-F_{n, x x}^{0} F_{n}=\left(F_{n}^{0} F_{n, x}-F_{n, x}^{0} F_{n}\right)_{x}=\left(E-E_{0}\right) P_{n, x}$, by Corollary A.1 we obtain

$$
\mu^{2}\left(F_{n}^{0}\right)^{2}-\mu_{0}^{2} F_{n}^{2}=\left(E-E_{0}\right)\left(\frac{F_{n} F_{n}^{0} P_{n, x}}{2}+F_{n}^{2}\left(F_{n}^{0}\right)^{2}-\frac{P_{n}\left(F_{n} F_{n, x}^{0}+F_{n, x} F_{n}^{0}\right)}{4}\right) .
$$

Now, let $\left(E_{0}, \mu_{0}\right)$ be a regular point of $\Gamma_{n}$ and $\mu_{0}=0$. In this case, we have that $R_{2 n+1}^{0}=$ $R_{2 n+1}\left(E_{0}\right)=0$ and $\partial_{E}\left(R_{2 n+1}\right)\left(E_{0}\right) \neq 0$, thus,

$$
\mu^{2}=R_{2 n+1}(E)=\left(E-E_{0}\right) M_{2 n},
$$

where $M_{2 n}(E)$ is a polynomial in $E$ of degree $2 n$ such that $M_{2 n}\left(E_{0}\right) \neq 0$.

Corollary A.3. Let $\left(E_{0}, \mu_{0}\right)$ be a regular point of $\Gamma_{n}$ and $\mu_{0}=0$. We have that

$$
\frac{M_{2 n}}{F_{n}}+\frac{\left(E-E_{0}\right) P_{n}^{2}}{4 F_{n}\left(F_{n}^{0}\right)^{2}}
$$

is a polynomial in $E$ of degree $n$, with $P_{n}$ the polynomial obtained in Corollary A.1 and $M_{2 n}$ the polynomial defined in (A.2).

Proof. We have

$$
\begin{aligned}
& M_{2 n}=\frac{\mu^{2}}{E-E_{0}}=\frac{F_{n} F_{n, x x}}{2\left(E-E_{0}\right)}-\frac{(u-E) F_{n}^{2}}{E-E_{0}}-\frac{F_{n, x}^{2}}{4\left(E-E_{0}\right)}, \\
& P_{n}^{2}=\frac{\left(F_{n}^{0} F_{n, x}-F_{n, x}^{0} F_{n}\right)^{2}}{\left(E-E_{0}\right)^{2}}=\frac{\left(F_{n}^{0}\right)^{2} F_{n, x}^{2}+\left(F_{n, x}^{0}\right)^{2} F_{n}^{2}-2 F_{n}^{0} F_{n} F_{n, x} F_{n, x}^{0}}{\left(E-E_{0}\right)^{2}} .
\end{aligned}
$$


We replace these expressions in the formula and we get

$$
\frac{M_{2 n}}{F_{n}}+\frac{\left(E-E_{0}\right) P_{n}^{2}}{4 F_{n}\left(F_{n}^{0}\right)^{2}}=\frac{2\left(F_{n}^{0}\right)^{2} F_{n, x x}-4(u-E)\left(F_{n}^{0}\right)^{2} F_{n}+\left(F_{n, x}^{0}\right)^{2} F_{n}-2 F_{n}^{0} F_{n, x}^{0} F_{n, x}}{4\left(E-E_{0}\right)\left(F_{n}^{0}\right)^{2}} .
$$

The numerator of this function is a polynomial in $E$ of degree $n+1$ and has a root in $E=E_{0}$ as can be easily verified replacing $E$ by $E_{0}$ :

$$
2\left(F_{n}^{0}\right)^{2} F_{n, x x}^{0}-4\left(u-E^{0}\right)\left(F_{n}^{0}\right)^{3}-\left(F_{n, x}^{0}\right)^{2} F_{n}^{0}=4 F_{n}^{0} \mu_{0}^{2}=0 .
$$

So, we get that

$$
2\left(F_{n}^{0}\right)^{2} F_{n, x x}-4(u-E)\left(F_{n}^{0}\right)^{2} F_{n}+\left(F_{n, x}^{0}\right)^{2} F_{n}-2 F_{n}^{0} F_{n, x}^{0} F_{n, x}=\left(E-E_{0}\right) Q_{n},
$$

where $Q_{n}$ denotes a polynomial in $E$ of degree $n$. Hence

$$
\frac{M_{2 n}}{F_{n}}+\frac{\left(E-E_{0}\right) P_{n}^{2}}{4 F_{n}\left(F_{n}^{0}\right)^{2}}=\frac{Q_{n}}{4\left(F_{n}^{0}\right)^{2}}
$$

and then the result follows.

Next, let $\left(E_{0}, \mu_{0}\right)$ be a singular point of $\Gamma_{n}$. In this case, $\mu_{0}=0, R_{2 n+1}^{0}=R_{2 n+1}\left(E_{0}\right)=0$ and $\partial_{E}\left(R_{2 n+1}\right)\left(E_{0}\right)=0$, thus,

$$
\mu^{2}=R_{2 n+1}(E)=\left(E-E_{0}\right)^{2} Z_{2 n-1},
$$

where $Z_{2 n-1}(E)$ is a polynomial in $E$ of degree $2 n-1$ such that $Z_{2 n-1}\left(E_{0}\right) \neq 0$.

Corollary A.4. Let $\left(E_{0}, \mu_{0}\right)$ be a singular point of $\Gamma_{n}$. We have that

$$
\frac{Z_{2 n-1}}{F_{n}}+\frac{P_{n}^{2}}{4 F_{n}\left(F_{n}^{0}\right)^{2}}
$$

is a polynomial in $E$ of degree $n-1$, with $P_{n}$ the polynomial obtained in Corollary A.1 and $Z_{2 n-1}$ the polynomial defined in (A.3).

Proof. It follows by an analogous computation to that of Corollary A.3.

\section{Acknowledgements}

We kindly thank all members of the Integrability Madrid Seminar for many fruitful discussions: P. Acosta-Humánez, D. Blázquez, J.A. Capitán, R. Hernández Heredero, A. PérezRaposo, J. Rojo Montijano and S. Rueda. Authors gratefully acknowledge the reviewers for their helpful comments and further references which resulted in an improvement of the preliminary manuscript.

\section{References}

[1] Adler M., Moser J., On a class of polynomials connected with the Korteweg-de Vries equation, Comm. Math. Phys. 61 (1978), 1-30.

[2] Airault H., McKean H.P., Moser J., Rational and elliptic solutions of the Korteweg-de Vries equation and a related many-body problem, Comm. Pure Appl. Math. 30 (1977), 95-148.

[3] Beardon A.F., Ng T.W., Parametrizations of algebraic curves, Ann. Acad. Sci. Fenn. Math. 31 (2006), $541-554$. 
[4] Braverman A., Etingof P., Gaitsgory D., Quantum integrable systems and differential Galois theory, Transform. Groups 2 (1997), 31-56, arXiv:alg-geom/9607012.

[5] Brezhnev Yu.V., What does integrability of finite-gap or soliton potentials mean?, Philos. Trans. R. Soc. Lond. Ser. A Math. Phys. Eng. Sci. 366 (2008), 923-945, arXiv:nlin.SI/0505003.

[6] Brezhnev Yu.V., Spectral/quadrature duality: Picard-Vessiot theory and finite-gap potentials, in Algebraic Aspects of Darboux Transformations, Quantum Integrable Systems and Supersymmetric Quantum Mechanics, Contemp. Math., Vol. 563, Amer. Math. Soc., Providence, RI, 2012, 1-31, arXiv:1011.1642.

[7] Brezhnev Yu.V., Elliptic solitons, Fuchsian equations, and algorithms, St. Petersburg Math. J. 24 (2013), $555-574$.

[8] Burchnall J.L., Chaundy T.W., Commutative ordinary differential operators, Proc. R. Soc. London Ser. A 118 (1928), 557-583.

[9] Clarkson P.A., Vortices and polynomials, Stud. Appl. Math. 123 (2009), 37-62, arXiv:0901.0139.

[10] Crum M.M., Associated Sturm-Liouville systems, Quart. J. Math. Oxford 6 (1955), 121-127.

[11] Darboux G., Sur une proposition relative aux équations linéaires, Comptes Rendus Acad. Sci. 94 (1882), $1456-1459$.

[12] Darboux G., Leçons sur la théorie générale des surfaces. II, Gauthier-Villars, Paris, 1889.

[13] Ehlers F., Knörrer H., An algebro-geometric interpretation of the Bäcklund-transformation for the Korteweg-de Vries equation, Comment. Math. Helv. 57 (1982), 1-10.

[14] Gel'fand I.M., Dikii L.A., Asymptotic properties of the resolvent of Sturm-Liouville equations and the algebra of Korteweg-de Vries equations, Russian Math. Surveys 30 (1975), no. 5, 77-113.

[15] Gesztesy F., Holden H., Soliton equations and their algebro-geometric solutions. Vol. I. (1+1)-dimensional continuous models, Cambridge Studies in Advanced Mathematics, Vol. 79, Cambridge University Press, Cambridge, 2003.

[16] Gu C., Hu H., Zhou Z., Darboux transformations in integrable systems. Theory and their applications to geometry, Mathematical Physics Studies, Vol. 26, Springer, Dordrecht, 2005.

[17] Hermite C., Sur l'équation de Lamé, Oeuvres of Charles Hermite. III, Gauthier-Villars, Paris, 1912.

[18] Jiménez S., Morales-Ruiz J.J., Sánchez-Cauce R., Zurro M.-A., Differential Galois theory and Darboux transformations for integrable systems, J. Geom. Phys. 115 (2017), 75-88.

[19] Marshall I., Semenov-Tian-Shansky M., Poisson groups and differential Galois theory of Schroedinger equation on the circle, Comm. Math. Phys. 284 (2008), 537-552, arXiv:0710.5456.

[20] Matveev V.B., Salle M.A., Darboux transformations and solitons, Springer Series in Nonlinear Dynamics, Springer-Verlag, Berlin, 1991.

[21] Morales-Ruiz J.J., Rueda S.L., Zurro M.-A., Algebro-geometric solitonic solutions and differential Galois theory, arXiv:1708.00431.

[22] Morales-Ruiz J.J., Rueda S.L., Zurro M.-A., Factorization of KdV Schrödinger operators using differential subresultants, arXiv:1902.05443.

[23] Olver P.J., Applications of Lie groups to differential equations, Graduate Texts in Mathematics, Vol. 107, Springer-Verlag, New York, 1986.

[24] Whittaker E.T., Watson G.N., A course of modern analysis, Cambridge Mathematical Library, Cambridge University Press, Cambridge, 1996.

[25] Wilson G., On the quasi-Hamiltonian formalism of the KdV equation, Phys. Lett. A 132 (1988), 445-450. 